\title{
DOUBLE HILBERT TRANSFORMS ALONG POLYNOMIAL SURFACES IN $\mathbf{R}^{3}$
}

\author{
SANJAY PATEL \\ Department of Mathematics, School of Sciences, Gujarat University, Navrangpura, \\ Ahmedabad-380009, India \\ e-mail: skpatel27@yahoo.com
}

(Received 24 September 2006; revised 22 December 2007; accepted 1 February 2008)

\begin{abstract}
We obtain a necessary and sufficient condition on a polynomial $P(s, t)$ so that the (global) double Hilbert transforms along polynomial surfaces $(s, t, P(s, t))$ in $\mathbf{R}^{3}$ are bounded on $L^{p}$ for $1<p<\infty$.
\end{abstract}

2000 Mathematics Subject Classification. 42B20.

1. Introduction. For $f \in \mathcal{S}$ (i.e., a Schwartz class function) define

$$
H_{l o c} f(x, y, z)=p \cdot v \cdot \int_{-1}^{1} \int_{-1}^{1} f(x-s, y-t, z-P(s, t)) \frac{\mathrm{d} s \mathrm{~d} t}{s t}
$$

where $P(s, t)$ is a real-valued polynomial in $s$ and $t$. Carbery, Wainger and Wright determined the necessary and sufficient condition on the polynomial $P$ so that $H_{l o c}$ is $L^{p}$ bounded for $1<p<\infty$. We state their result.

Theorem 1.1. For any $p, 1<p<\infty$,

$$
\left\|H_{l o c} f\right\|_{L^{p}} \leq A_{p}\|f\|_{L^{p}}
$$

iff for each $(m, n)$ that is a corner point of the Newton diagram corresponding to $P$, at least one of $m$ and $n$ is even.

In this paper, we determine a necessary and sufficient condition on $P$ so that the (global) double Hilbert transform defined by

$$
H f(x, y, z)=p \cdot v \cdot \int_{-\infty}^{\infty} \int_{-\infty}^{\infty} f(x-s, y-t, z-P(s, t)) \frac{\mathrm{d} s \mathrm{~d} t}{s t}
$$

is bounded on $L^{p}, 1<p<\infty$. The operator is defined for $f \in \mathcal{S}$ by integrating where $\epsilon^{\prime} \leq|s| \leq R^{\prime}, \epsilon \leq|t| \leq R$, and then, taking the limits as $\epsilon, \epsilon^{\prime} \rightarrow 0$ and $R, R^{\prime} \rightarrow+\infty$. We now state the main result of this paper.

Let $P(s, t)=\sum_{(m, n) \in \Lambda} a_{m, n} s^{m} t^{n}$ be a polynomial with real coefficients such that $P(0,0)=0, \nabla P(0,0)=0$ and where $\Lambda$ is indexing the set of lattice points $(m, n) \in \mathbf{Z}^{2}$ such that $a_{m, n} \neq 0$.

Let $\mathcal{C}$ be the closed convex hull of $\Lambda$ in $\mathbf{R}^{2}$ and

$$
\mathcal{D}=\{(m, n) \in \Lambda:(m, n) \text { is a corner point (vertex) of } \mathcal{C}\}
$$


Theorem 1.2 Main Theorem. For any $p, 1<p<\infty$,

$$
\|H f\|_{L^{p}} \leq A_{p}\|f\|_{L^{p}}
$$

if and only if for each $(m, n) \in \mathcal{D}$, at least one of $m$ and $n$ is even and furthermore, if any (extended) edge of $\mathcal{C}$ passes through the origin (there are at most two such edges), then every point of $\Lambda$ on that edge must have at least one even coordinate.

We note that the $L^{p}, 1<p<\infty$, boundedness of the maximal functions associated to $H$ or $H_{l o c}$ is not an issue here. This follows easily (without any condition on $P$ and also independent of the coefficients of $P$ ) from [4, Theorem 7.1] and de Leeuw's theorem.

We now give the organisation of our paper. In Section 2, we discuss a few known results about the maximal and singular integral operators and then begin with the proof of sufficiency part of our main theorem. To do this, we initially assume that none of the edges of $\mathcal{C}$ pass through the origin and later adapt this proof to the other case. In Section 3, we decompose the operator $H$ by splitting the region of integration where we can replace $P(s, t)$ by one of the monomials corresponding to a corner point of the convex hull $\mathcal{C}$, with an error that is bounded on $L^{p}$. In Section 4 , we define the approximating operators and obtain a few preliminary estimates. In Section 5, we prove the $L^{p}$ boundedness of the difference (error) between $H$ and the approximating operators using certain results of Duoandikoetxea and Rubio de Francia that we quote in Section 2. In Section 6, we prove the $L^{p}$ boundedness of the approximating operators using a result of Ricci and Stein. In Section 7, we discuss the proof of the sufficiency part assuming that $\mathcal{C}$ has an edge through the origin. In Section 8, we discuss the proof of the sufficiency part in the particular cases excluded from the outset. Finally in Section 9, we discuss the necessary part of the hypothesis of the main theorem.

Apart from some technical difficulties in extending the result of [2], the main difficulty is to tackle the case of an edge through the origin. The method of decomposing the operator $H$ into finitely many operators and introducing approximating operators in Sections 3 and 4 is almost the same as in [2]. Our method differs from [2] while handling the difference between $H$ and the approximating operators in Section 5. In [2], this has been done using the standard Littlewood-Paley theory that do not seem to work here.

One may wonder what happens to Theorem 1.2 if $P$ is assumed to be a real-analytic function? But the assumption that $P$ is a polynomial helps significantly while proving estimate (12) of Lemma 4.2 and while determining a positive number $\sigma$ in estimate (13) of Lemma 5.2. If one can achieve this with a real-analytic $P$, then the assumption that $P$ is a polynomial in Theorem 1.2 could possibly be replaced by the assumption that $P$ is a real-analytic function.

2. A few known results. Here, we shall state two theorems (with a different formulation) due to J. Duoandikoetxea and J. L. Rubio de Francia [3] and a result that is due to Ricci and Stein [4]. We shall need these results while proving our main theorem. We begin with some notation and terminology.

- Let $\mathbf{R}^{n}=\mathbf{R}^{m} \times \mathbf{R}^{n-m}$ with $1 \leq m<n$, and write $x \in \mathbf{R}^{n}$ in the form $x=\left(x^{0}, \bar{x}\right), x^{0} \in$ $\mathbf{R}^{m}, \bar{x} \in \mathbf{R}^{n-m}$. Then, given a finite measure $\omega$ on $\mathbf{R}^{n}$, we define another measure $\omega^{(0)}$ on $\mathbf{R}^{m}$ as $\omega^{(0)}(E)=\omega\left(E \times \mathbf{R}^{n-m}\right)$ for every Borel subset $E$ of $\mathbf{R}^{m}$; in terms of Fourier transforms, this means $\widehat{\omega^{(0)}}\left(\zeta^{0}\right)=\hat{\omega}\left(\zeta^{0}, 0\right)$. Also, if $f$ is a Schwartz function in $\mathbf{R}^{m}$, 
then

$$
<f, \omega^{(0)}>=\int f\left(x^{0}\right) \mathrm{d} \omega\left(x^{0}, \bar{x}\right)
$$

- We use the dilations given by the family of matrices $\{A(t)\}_{t \geq 0}$ satisfying $A(s t)=$ $A(s) A(t)$ and the Rivière condition. That is, for each $t>0, A(t) \in G L(n, \mathbf{R})$, and

$$
\left\|A(t) A^{-1}(s t)\right\| \leq C / s^{\epsilon}
$$

for all $s>1$, all $t>0$, some $\epsilon>0$ and some $C \geq 1$. We also assume that the norm is the $l_{n}^{2}$-operator norm. For example,

$$
A(t)=\left(\begin{array}{lr}
t^{\sigma_{1}} & 0 \\
0 & t^{\sigma_{2}}
\end{array}\right)
$$

on $\mathbf{R}^{2}$ are easily seen to satisfy the Rivière condition with $\epsilon=\min \left(\sigma_{1}, \sigma_{2}\right)$ if $\sigma_{1}$ and $\sigma_{2}$ are positive. It is not hard to see that (1) holds if and only if $A(t)=\exp \{B \log t\}$ for some real matix $B$ each of whose eigen value has a positive real part.

With these definitions, we have the following two theorems due to $\mathrm{J}$. Duoandikoetxea and J. L. Rubio de Francia.

THEOREM 2.1. Let $\left\{\omega_{k}\right\}_{k=-\infty}^{\infty}$ be probability measures in $\mathbf{R}^{n}$ such that

$$
\begin{gathered}
\left|\widehat{\omega}_{k}\left(\zeta^{0}, \bar{\zeta}\right)-\widehat{\omega}_{k}\left(\zeta^{0}, 0\right)\right| \leq C\left|A\left(2^{-k}\right) \bar{\zeta}\right|^{\alpha} \\
\left|\widehat{\omega}_{k}\left(\zeta^{0}, \bar{\zeta}\right)\right| \leq C\left|A\left(2^{-k}\right) \bar{\zeta}\right|^{-\alpha}
\end{gathered}
$$

where $\zeta=\left(\zeta^{0}, \bar{\zeta}\right) \in \mathbf{R}^{n}, \zeta^{0} \in \mathbf{R}^{m}, \bar{\zeta} \in \mathbf{R}^{n-m}$ and $\alpha>0$. Suppose that $M^{0} g\left(x^{0}\right)=$ $\sup _{k}\left|\omega_{k}^{(0)} * g\left(x^{0}\right)\right|$ is a bounded operator on $L^{p}\left(\mathbf{R}^{m}\right)$ for all $p>1$. Then, $M f(x)=$ $\sup _{k}\left|\omega_{k} * f(x)\right|$ is also bounded on $L^{p}\left(\mathbf{R}^{n}\right)$ for all $p>1$.

THEOREM 2.2. Let $\left\{\rho_{k}\right\}_{k=-\infty}^{\infty}$ be measures such that $\left\|\rho_{k}\right\| \leq 1$ and

$$
\left|\widehat{\rho}_{k}\left(\zeta^{0}, \bar{\zeta}\right)\right| \leq C \min \left(\left|A\left(2^{-k}\right) \bar{\zeta}\right|,\left|A\left(2^{-k}\right) \bar{\zeta}\right|^{-1}\right)^{\alpha}
$$

where $\zeta=\left(\zeta^{0}, \bar{\zeta}\right) \in \mathbf{R}^{n}, \zeta^{0} \in \mathbf{R}^{m}, \bar{\zeta} \in \mathbf{R}^{n-m}$ and $\alpha>0$. Suppose that $\rho^{*} f=\sup _{k}|| \rho_{k}|* f|$ is bounded on $L^{q}\left(\mathbf{R}^{n}\right)$. Then, $T f=\sum_{-\infty}^{\infty} \rho_{k} * f$ and $g(f)=\left(\sum_{k}\left|\rho_{k} * f\right|^{2}\right)^{1 / 2}$ are bounded on $L^{p}\left(\mathbf{R}^{n}\right)$ for $\left|\frac{1}{p}-\frac{1}{2}\right|<\frac{1}{2 q}$.

Moreover, the $L^{p}-L^{p}$ norm of the operator $M$ in Theorem 2.1 does not depend on the family $\left\{\omega_{k}\right\}_{k=-\infty}^{\infty}$ in the sense that it can be controlled by the constant ' $C$ ' of (2) and (3), the constant of Rivière condition and the $L^{p}-L^{p}$ norm of the operator $M^{0}$. Similarly, the $L^{p}-L^{p}$ norm of the operator $T$ and $g$ in Theorem 2.2 can be controlled by the constant ' $C$ ' of (4), the constant of Rivière condition and the $L^{q}-L^{q}$ norm of the operator $\rho^{*}$.

Next we state a theorem that is a special case of a general result of Ricci and Stein [4, Theorem 5.1]. For each $I=(p, q) \in \mathbf{Z}^{2}$, let $\mu^{(I)}$ denote the associated finite measure 
supported in the unit cube of $\mathbf{R}^{3}$. Define the dilated $\mu_{I}^{(I)}$ of $\mu^{(I)}$ by

$$
\int_{\mathbf{R}^{3}} f \mathrm{~d} \mu_{I}^{(I)}=\int_{\mathbf{R}^{3}} f\left(2^{-p} x, 2^{-q} y, 2^{-p m} 2^{-q n} z\right) \mathrm{d} \mu^{(I)}(x, y, z),
$$

where $m$ and $n$ are fixed integers. Then, we have the following result.

Theorem 2.3 (Ricci and Stein). Suppose

(i) $\left|\widehat{\mu^{(I)}}(\xi)\right| \leq C(1+|\xi|)^{-\epsilon}$ for some $C, \epsilon>0$;

(ii) $\left|\mu^{(I)}\right| \leq \sigma \in \mathcal{B}\left(\mathbf{R}^{n}\right)$ (finite Borel measure);

(iii) $\widehat{\mu^{(I)}}\left(\lambda e_{j}\right)=0$ for all $\lambda \in \mathbf{R}$ and $1 \leq j \leq 3$, where $\left\{e_{j}\right\}$ form the canonical basis for $\mathbf{R}^{3}$. Furthermore, if either $n$ or $m$ is zero, then $\widehat{\mu^{(I)}}\left(\xi_{1}, 0, \xi_{3}\right) \equiv 0$ or $\widehat{\mu^{(I)}}\left(0, \xi_{2}, \xi_{3}\right) \equiv$ 0 , respectively.

Then, convolution with the kernel $K=\sum_{I \in \mathbf{Z}^{2}} \mu_{I}^{(I)}$ is bounded on $L^{p}\left(\mathbf{R}^{3}\right), 1<p<\infty$.

We shall use the above theorems in the proof of our main theorem that we give now.

Back to main theorem (Theorem 1.2). We begin with a few remarks about the statement of our main theorem.

- The constant $A_{p}$ of Theorem 1.2 depends on $p$ as well as the polynomial $P$.

- Throughout 'edge of $\mathcal{C}$ ' means an extended edge.

- Throughout the proof of the theorem, ' $C$ ' denotes a general constant.

We first give the proof of the sufficiency part of the main theorem. That is, we assume that every corner point of $\mathcal{C}$ and also every point of $\Lambda$ on an edge of $\mathcal{C}$ through the origin (if it exists) have at least one even coordinate. Under this assumption, we shall show that $H$ is $L^{p}$ bounded for $1<p<\infty$. For this, we shall initially assume that $\mathcal{C}$ has at least three corner points so that the interior of $\mathcal{C}$ is non-empty. The proof of the other particular cases will be discussed later.

We initially assume that no edge of $\mathcal{C}$ passes through the origin and later adapt this proof to the other case.

3. Decomposition of the operator $\boldsymbol{H}$. We choose a $C^{\infty}$ function $\psi(s)$ defined on the real line that is odd, non-negative for $s \geq 0$, supported in $1 / 2 \leq|s| \leq 2$ and such that

$$
\sum_{p \in \mathbf{Z}} 2^{p} \psi\left(2^{p} s\right)=1 / s
$$

We then define

$$
H_{p, q} f(x, y, z)=2^{p+q} \iint f(x-s, y-t, z-P(s, t)) \psi\left(2^{p} s\right) \psi\left(2^{q} t\right) \mathrm{d} s \mathrm{~d} t .
$$

Thus,

$$
H=\sum_{(p, q) \in \mathbf{Z} \times \mathbf{Z}} H_{p, q}
$$


We next consider the orientation of the boundary of $\mathcal{C}$ to be anticlockwise and assume that it has $r(\geq 3)$ corner points namely $v_{1}, v_{2}, \ldots, v_{r}$ in the anticlockwise order. We let $v_{j}=\left(m_{j}, n_{j}\right)$ for $1 \leq j \leq r$.

Then, $\mathcal{C}$ has $r$ edges that we denote by the vectors $\bar{v}_{j} v_{j+1}=v_{j+1}-v_{j}$ for $1 \leq j \leq r$, considering the point $v_{r+1}$ the same as the corner point $v_{1}$. For $1 \leq j \leq r$, let $\bar{n}_{j}=\left(n_{j}^{1}, n_{j}^{2}\right)$ denote the (inward) normal vector to the edge $\bar{v}_{j} v_{j+1}$ of $\mathcal{C}$. Also, we choose $\bar{n}_{j}$ such that $n_{j}^{1}$ and $n_{j}^{2}$ are integers. (Such a choice is not unique but the choice does not matter here.) Since $\mathcal{C}$ is the closed convex hull of $\Lambda$, geometrically it is now easy to see that

$$
\left(v-v_{j}\right) \cdot \bar{n}_{j-1} \geq 0 \quad \text { and } \quad\left(v-v_{j}\right) \cdot \bar{n}_{j} \geq 0 \quad \text { for all } v \in \Lambda .
$$

For $1 \leq j \leq r$, define

$$
\begin{aligned}
T(j) & =\left\{(p, q) \in \mathbf{Z} \times \mathbf{Z}:(p, q) \cdot\left(v-v_{j}\right)>0 \quad \text { for all } v=(m, n) \in \Lambda \backslash\left\{v_{j}\right\}\right\} \\
& =\bigcap_{v \in \Lambda \backslash\left\{v_{j}\right\}}\left\{(p, q) \in \mathbf{Z} \times \mathbf{Z}:(p, q) \cdot\left(v-v_{j}\right)>0\right\} .
\end{aligned}
$$

In other words, $T(j)$ is the intersection of various half-planes.

LEMMA 3.1. For $1 \leq j \leq r$,

$$
T(j)=\left\{(p, q) \in \mathbf{Z} \times \mathbf{Z}:(p, q)=\alpha \bar{n}_{j-1}+\beta \bar{n}_{j} \text { for some positive reals } \alpha \text { and } \beta\right\}
$$

where $\bar{n}_{0}$ is considered to be the same as $\bar{n}_{r}$.

Proof. Since $v_{j}$ is one of the corner points of $\mathcal{C}$, the edges ${\overline{v_{j-1}}}_{j}$ and $\bar{v}_{j} v_{j+1}$ cannot be parallel and so the normals $\bar{n}_{j-1}$ and $\bar{n}_{j}$ are linearly independent directions. Thus, we can write $(p, q) \in \mathbf{Z} \times \mathbf{Z}$ as

$$
(p, q)=\alpha \bar{n}_{j-1}+\beta \bar{n}_{j} \text { for some real numbers } \alpha \text { and } \beta .
$$

We need to show that $(p, q) \in T(j)$ if and only if $\alpha, \beta>0$.

First suppose that $(p, q) \in T(j)$. Then,

$$
(p, q) \cdot\left(v-v_{j}\right)>0 \quad \text { for all } v \in \Lambda \backslash\left\{v_{j}\right\} .
$$

In particular,

$$
(p, q) \cdot\left(v_{j+1}-v_{j}\right)>0
$$

Therefore,

$$
\left(\alpha \bar{n}_{j-1}+\beta \bar{n}_{j}\right) \cdot\left(v_{j+1}-v_{j}\right)>0 .
$$

But $\bar{n}_{j}$ being normal to the edge ${\overline{v_{j}}}_{j+1}=v_{j+1}-v_{j}$,

$$
\bar{n}_{j} \cdot\left(v_{j+1}-v_{j}\right)=0 .
$$

So we have

$$
\alpha\left(\bar{n}_{j-1} \cdot\left(v_{j+1}-v_{j}\right)\right)>0
$$


which implies $\alpha>0$ due to (6). Similarly, choosing $v=v_{j-1}$ gives $\beta>0$. Conversely, if $\alpha>0$ and $\beta>0$, then for any $v \in \Lambda \backslash\left\{v_{j}\right\}$,

$$
(p, q) \cdot\left(v-v_{j}\right)=\alpha\left(\bar{n}_{j-1} \cdot\left(v-v_{j}\right)\right)+\beta\left(\bar{n}_{j} \cdot\left(v-v_{j}\right)\right)
$$

But by (6),

$$
\bar{n}_{j-1} \cdot\left(v-v_{j}\right) \geq 0 \quad \text { and } \quad \bar{n}_{j} \cdot\left(v-v_{j}\right) \geq 0 \quad \text { for all } v \in \Lambda \backslash\left\{v_{j}\right\} .
$$

Also, for a given $v, \bar{n}_{j-1} \cdot\left(v-v_{j}\right)$ and $\bar{n}_{j} \cdot\left(v-v_{j}\right)$ both cannot be simultaneously zero as $\bar{n}_{j}$ and $\bar{n}_{j-1}$ are non-parallel. So,

$$
(p, q) \cdot\left(v-v_{j}\right)>0 \quad \text { if } \alpha>0 \text { and } \beta>0 .
$$

This completes the proof of Lemma 3.1.

We next show that these sets are pairwise disjoint.

LEMMA 3.2. $T(j) \bigcap T(k)=\emptyset$ for $1 \leq j<k \leq r$.

Proof. Suppose $(p, q) \in T(j) \bigcap T(k)$. Then,

$$
(p, q) \cdot\left(v-v_{j}\right)>0 \quad \text { for all } v \in \Lambda \backslash\left\{v_{j}\right\}
$$

and

$$
(p, q) \cdot\left(v-v_{k}\right)>0 \quad \text { for all } v \in \Lambda \backslash\left\{v_{k}\right\} .
$$

In particular,

$$
(p, q) \cdot\left(v_{k}-v_{j}\right)>0 \quad \text { and } \quad(p, q) \cdot\left(v_{j}-v_{k}\right)>0
$$

which is not possible. This concludes Lemma 3.2.

Now Lemma 3.1 asserts that

$$
\begin{aligned}
T(j) & =\left\{(p, q) \in \mathbf{Z} \times \mathbf{Z}:(p, q) \cdot\left(v-v_{j}\right)>0 \text { for all } v \in \Lambda \backslash\left\{v_{j}\right\}\right\} \\
& =\left\{(p, q) \in \mathbf{Z} \times \mathbf{Z}:(p, q)=\alpha \bar{n}_{j-1}+\beta \bar{n}_{j} \text { for some } \alpha>0 \text { and } \beta>0\right\} .
\end{aligned}
$$

The second formulation of $T(j)$ precisely means that $T(j)$ consists of those points of $\mathbf{Z} \times \mathbf{Z}$ that are within the infinite triangle (or say cone centred at origin) whose boundaries are the lines (rays) in the direction of $\bar{n}_{j-1}$ and $\bar{n}_{j}$. Thus, if we define

$$
\begin{aligned}
Z(j) & =T(j) \cup\left\{(p, q) \in \mathbf{Z} \times \mathbf{Z}:(p, q)=\alpha \bar{n}_{j-1} ; \alpha>0\right\} \\
& =\left\{(p, q) \in \mathbf{Z} \times \mathbf{Z}:(p, q)=\alpha \bar{n}_{j-1}+\beta \bar{n}_{j} ; \alpha>0, \beta \geq 0\right\}
\end{aligned}
$$

then it follows from Lemma 3.2 that

$$
Z(j) \bigcap Z(k)=\emptyset \quad \text { for } 1 \leq j<k \leq r
$$

and geometrically, it is easy to see that

$$
\bigcup_{j=1}^{r} Z(j)=\mathbf{Z} \times \mathbf{Z} \backslash\{(0,0)\} .
$$


We note that for $(p, q) \in Z(j),(p, q) \cdot\left(v-v_{j}\right) \geq 0$ for all $v \in \Lambda$ by (6). Here, the strict inequality may not be achieved even for $v \neq v_{j}$.

We now try to determine $\alpha$ and $\beta$ explicitly for a given $(p, q) \in Z(j)$. We have

$$
\left(\begin{array}{l}
p \\
q
\end{array}\right)=\left(\begin{array}{c}
\alpha n_{j-1}^{1}+\beta n_{j}^{1} \\
\alpha n_{j-1}^{2}+\beta n_{j}^{2}
\end{array}\right)=\left(\begin{array}{cc}
n_{j-1}^{1} & n_{j}^{1} \\
n_{j-1}^{2} & n_{j}^{2}
\end{array}\right)\left(\begin{array}{c}
\alpha \\
\beta
\end{array}\right) .
$$

The determinant $d$ of the above matrix on the right is non-zero since $\bar{n}_{j}$ and $\bar{n}_{j-1}$ are non-parallel. Thus,

$$
\left(\begin{array}{l}
\alpha \\
\beta
\end{array}\right)=\frac{1}{d}\left(\begin{array}{cc}
n_{j}^{2} & -n_{j}^{1} \\
-n_{j-1}^{2} & n_{j-1}^{1}
\end{array}\right)\left(\begin{array}{c}
p \\
q
\end{array}\right)=\left(\begin{array}{c}
\frac{p n_{j}^{2}-q n_{j}^{1}}{d} \\
\frac{-p n_{j-1}^{2}+q n_{j-1}^{1}}{d}
\end{array}\right) .
$$

Since we have chosen the components of $\bar{n}_{j-1}$ and $\bar{n}_{j}$ to be integers, we can write $\alpha=t / d$ and $\beta=l / d$ for some integers $t, l$ and $d$. Also, we know that $\alpha>0$ and $\beta \geq 0$ and so $t, l$ and $d$ are all either non-positive or non-negative. But since we have chosen the orientation of $\mathcal{C}$ to be anticlockwise, it is not hard to see that $d>0$ and so $t, l$ and $d$ are all non-negative integers. Thus, we have

$$
\begin{aligned}
Z(j)=\left\{(p, q) \in \mathbf{Z} \times \mathbf{Z}:(p, q)=\frac{t}{d} \bar{n}_{j-1}+\frac{l}{d} \bar{n}_{j} ; t \in \mathbf{N}, l \in \mathbf{N}^{\star}\right. \\
\text { where } \left.\quad d=n_{j-1}^{1} n_{j}^{2}-n_{j-1}^{2} n_{j}^{1}\right\}
\end{aligned}
$$

and where $\mathbf{N}^{\star}=\mathbf{N} \cup\{0\}$. We note that $d$ depends on the coordinates of $\bar{n}_{j-1}$ and $\bar{n}_{j}$, but for fixed $j, d$ is fixed. Now since we do not want to leave the point $(p, q)=(0,0)$, we include it in the set $Z(1)$. Thus,

$$
\bigcup_{j=1}^{r} Z(j)=\mathbf{Z} \times \mathbf{Z}
$$

We shall use this geometry to split our main operator

$$
H=\sum_{(p, q) \in \mathbf{Z} \times \mathbf{Z}} H_{p, q} .
$$

For $1 \leq j \leq r$, define

$$
M(j)=\sum_{(p, q) \in Z(j)} H_{p, q}
$$

Then from (7), it follows that

$$
H=\sum_{j=1}^{r} M(j)
$$


It is now sufficient to prove that $M(j)$ is bounded on $L^{p}\left(\mathbf{R}^{3}\right)$ (with bounds allowed to depend on $j$ ) for $1<p<\infty$. For this purpose, we introduce the approximating operators.

4. The approximating operators and a few preliminary estimates. Set

$$
S_{p, q} f(x, y, z)=2^{p+q} \iint f\left(x-s, y-t, z-a_{m_{j}, n_{j}} s^{m_{j}} t^{n_{j}}\right) \psi\left(2^{p} s\right) \psi\left(2^{q} t\right) \mathrm{d} s \mathrm{~d} t
$$

for $(p, q)$ in $Z(j)$, and

$$
Q(j)=\sum_{(p, q) \in Z(j)} S_{p, q} .
$$

Let $E(j)=M(j)-Q(j)$. As we shall see, $L^{p}$ boundedness of $Q(j)$ follows from the result of Ricci and Stein, and so, our main task is to prove the following proposition.

Proposition 4.1. For $1<p<\infty$,

$$
\|E(j)\|_{L^{p}} \leq A(p, j)\|f\|_{L^{p}} .
$$
Then,

Proof. Let $m_{p, q}$ denote the multiplier corresponding to $H_{p, q}-S_{p, q}$ for $(p, q)$ in $Z(j)$.

$$
\begin{aligned}
m_{p, q}(\xi, \eta, \gamma)= & 2^{p+q} \iint \exp (i \xi s+i \eta t)[\exp (i \gamma P(s, t)) \\
& \left.-\exp \left(i \gamma a_{m_{j}, n_{j}} s^{m_{j}} t^{n_{j}}\right)\right] \psi\left(2^{p} s\right) \psi\left(2^{q} t\right) \mathrm{d} s \mathrm{~d} t
\end{aligned}
$$

which after the change of variables is the same as

$$
\begin{aligned}
m_{p, q}(\xi, \eta, \gamma)= & \iint \exp \left(i \xi 2^{-p} s+i \eta 2^{-q} t\right)\left[\exp \left(i \gamma P\left(2^{-p} s, 2^{-q} t\right)\right)\right. \\
& \left.-\exp \left(i \gamma 2^{-p m_{j}} 2^{-q n_{j}} a_{m_{j}, n_{j}} s^{m_{j}} t^{n_{j}}\right)\right] \psi(s) \psi(t) \mathrm{d} s \mathrm{~d} t
\end{aligned}
$$

We write

$$
\begin{aligned}
P\left(2^{-p} s, 2^{-q} t\right) & =\sum_{(m, n) \in \Lambda} a_{m, n} 2^{-p m} 2^{-q n} s^{m} t^{n} \\
& =2^{-\left(p m_{j}+q n_{j}\right)} \sum_{(m, n) \in \Lambda} a_{m, n} 2^{-\left((p m+q n)-\left(p m_{j}+q n_{j}\right)\right)} s^{m} t^{n} \\
& =2^{-(p, q) \cdot v_{j}} \tilde{P}_{j}(s, t)
\end{aligned}
$$

where

$$
\tilde{P}_{j}(s, t)=\sum_{v=(m, n) \in \Lambda} a_{m, n} 2^{-(p, q) \cdot\left(v-v_{j}\right)} s^{m} t^{n}
$$

(We shall always keep in mind that $\tilde{P}_{j}$ depends on the choice of the pair $(p, q) \in Z(j)$. But later we shall also see that $\tilde{P}_{j}$ enjoys certain uniformity properties in $\left.(p, q) \in Z(j)\right)$. 
So,

$$
\begin{aligned}
m_{p, q}(\xi, \eta, \gamma)= & \iint \exp \left(i \xi 2^{-p} s+i \eta 2^{-q} t\right)\left[\exp \left(i \gamma 2^{-(p, q) \cdot v_{j}} \tilde{P}_{j}(s, t)\right)\right. \\
& \left.-\exp \left(i \gamma 2^{-(p, q) \cdot v_{j}} a_{m_{j}, n_{j}} s^{m_{j}} t^{n_{j}}\right)\right] \psi(s) \psi(t) \mathrm{d} s \mathrm{~d} t
\end{aligned}
$$

We then have the following estimates for $m_{p, q}$.

LEMMA 4.2. For $(p, q)$ in $Z(j)$,

$$
\left|m_{p, q}(\xi, \eta, \gamma)\right| \leq A|\gamma| 2^{-(p, q) \cdot v_{j}}
$$

and

$$
\left|m_{p, q}(\xi, \eta, \gamma)\right| \leq \frac{A}{\left(|\xi| 2^{-p}+|\eta| 2^{-q}+|\gamma| 2^{-(p, q) \cdot v_{j}}\right)^{\epsilon}}
$$

for some $\epsilon>0$. The constant $A$ is independent of $p$ and $q$ but may depend on $v_{j}$ and $P$.

Proof. For $(p, q) \in Z(j)$, we know that $(p, q) \cdot\left(v-v_{j}\right) \geq 0$ and so estimate (11) follows from (9) and (10) and the mean value theorem.

For estimate (12), we treat each term of $m_{p, q}$ individually. Since $(p, q) \cdot\left(v-v_{j}\right) \geq 0$ for all $(p, q) \in Z(j)$,

$$
\tilde{P}_{j}(s, t)=\sum_{(m, n) \in \Lambda} a_{m, n} 2^{-(p, q) \cdot\left(v-v_{j}\right)} s^{m} t^{n}=\sum_{(m, n) \in \Lambda} b_{m, n} s^{m} t^{n}
$$

is uniformly (in terms of $(p, q) \in Z(j)$ ) in any $C^{k}$ class $^{\dagger}$ (for $|s|,|t| \leq 2$ ) and satisfies $\sum\left|b_{m, n}\right| \geq\left|a_{m_{j}, n_{j}}\right|$. Also since the 'norms'

$$
\sum\left|b_{m, n}\right| \quad \text { and } \quad \max _{1 \leq|\alpha| \leq d_{0}} \inf _{|s|,|t| \leq 2}\left|\partial^{\alpha} \tilde{P}_{j}(s, t)\right|
$$

are equivalent on the finite-dimensional vector space of non-constant polynomials of degree at most $d_{0}$, we see that some derivative of $\tilde{P}_{j}$ is uniformly bounded below. (We note that the latter is not really a norm because it does not satisfy the triangle inequality for a norm. However it can be verified that we do not need the triangle inequality for the latter to show that both are comparable. See for instance, the proof of [1, Corollary 7.3]). Now $\nabla P_{j}(0,0)=0$ (hypothesis) and so if $\partial^{\alpha} \tilde{P}_{j}$ is uniformly bounded below, then $|\alpha| \geq 2$. Let $m_{p, q}=m_{p, q}^{1}-m_{p, q}^{2}$ where

$$
\begin{aligned}
m_{p, q}^{1}(\xi, \eta, \gamma) & =\iint \exp \left(i \xi 2^{-p} s+i \eta 2^{-q} t+i \gamma 2^{-(p, q) \cdot\left(v-v_{j}\right)} \tilde{P}_{j}(s, t)\right) \psi(s) \psi(t) \mathrm{d} s \mathrm{~d} t \\
& =\iint \exp \left[i \lambda\left(\omega \cdot\left(s, t, \tilde{P}_{j}(s, t)\right)\right] \psi(s) \psi(t) \mathrm{d} s \mathrm{~d} t\right.
\end{aligned}
$$

$\omega=\left(w_{1}, w_{2}, w_{3}\right)$ is the unit vector $\frac{1}{\left|\left(\xi 2^{-p}, \eta 2^{-q}, \gamma 2^{-(p, q) \cdot\left(v-v_{j}\right)}\right)\right|}\left(\xi 2^{-p}, \eta 2^{-q}, \gamma 2^{-(p, q) \cdot\left(v-v_{j}\right)}\right)$ and $\lambda=\left|\left(\xi 2^{-p}, \eta 2^{-q}, \gamma 2^{-(p, q) \cdot\left(v-v_{j}\right)}\right)\right|$.

$\bar{\dagger} \bar{A}$ function is in the $C^{k}$ class if all of its mixed derivatives up to the order $k$ are bounded above. 
Next we set $\Phi_{j}(s, t)=\omega \cdot\left(s, t, \tilde{P}_{j}(s, t)\right)$. Then,

$$
\begin{aligned}
& \frac{\partial \Phi_{j}}{\partial s}=w_{1}+w_{3} \frac{\partial \tilde{P}_{j}}{\partial s} \\
& \frac{\partial \Phi_{j}}{\partial t}=w_{2}+w_{3} \frac{\partial \tilde{P}_{j}}{\partial t}
\end{aligned}
$$

and

$$
\partial^{\alpha} \Phi_{j}=w_{3} \partial^{\alpha} \tilde{P}_{j} \quad \text { for }|\alpha| \geq 2
$$

Since, $\omega$ is a unit vector, $\partial^{\alpha} \tilde{P}_{j}$ is uniformly bounded below for some $\alpha$ with $|\alpha| \geq 2$, and $\tilde{P}_{j}$ is uniformly in any $C^{k}$ class; it follows that $\partial^{\alpha} \Phi_{j}$ is uniformly bounded below for some $\alpha$. Also, $\tilde{P}_{j}$ being uniformly in any $C^{k}$ class, $\Phi_{j}$ is uniformly in any $C^{k}$ class and so estimate (12) (with $A$ independent of $(p, q) \in Z(j))$ for $m_{p, q}^{1}$ follows from van der Corput's lemma in higher dimensions [5, Chapter VIII, Proposition 5]. The same estimate also holds for $m_{p, q}^{2}$ because it is just the special case of $m_{p, q}^{1}$, that is, when $\tilde{P}_{j}$ is a monomial. This completes the proof of Lemma 4.2.

We shall use the estimates of Lemma 4.2 to show that the multiplier of $E(j)$ is an $L^{p}$ multiplier on $\mathbf{R}^{3}$, and in particular, it is a bounded function. For this purpose, we further split our operator $E(j)$ by splitting $Z(j)$ into $Z_{1}(j)$ and $Z_{2}(j)$ where

$$
\begin{aligned}
& Z_{1}(j)=\left\{(p, q) \in Z(j):(p, q)=\frac{k+N}{d} \bar{n}_{j-1}+\frac{N}{d} \bar{n}_{j} ; k \in \mathbf{N} \text { and } N \in \mathbf{N}^{\star}\right\} \\
& Z_{2}(j)=\left\{(p, q) \in Z(j):(p, q)=\frac{N}{d} \bar{n}_{j-1}+\frac{k+N}{d} \bar{n}_{j} ; N \in \mathbf{N} \text { and } k \in \mathbf{N}^{\star}\right\} .
\end{aligned}
$$

Since $\bar{n}_{j-1}$ and $\bar{n}_{j}$ are linearly independent, it is clear that $Z(j)$ is the disjoint union of $Z_{1}(j)$ and $Z_{2}(j)$. Also when $v_{j}=v_{1}$, we add the point $(0,0)$ to either $Z_{1}(1)$ or $Z_{2}(1)$. We assume it is in $Z_{1}(1)$. We now split $E(j)$ as $E(j)=E_{1}(j)+E_{2}(j)$ where

$$
E_{1}(j)=\sum_{(p, q) \in Z_{1}(j)}\left(H_{p, q}-S_{p, q}\right)=M_{1}(j)-Q_{1}(j)
$$

and

$$
E_{2}(j)=\sum_{(p, q) \in Z_{2}(j)}\left(H_{p, q}-S_{p, q}\right)=M_{2}(j)-Q_{2}(j) .
$$

To prove Proposition 4.1, it suffices to prove the next proposition.

Proposition 4.3. For $1 \leq j \leq r$ and $1<p<\infty$,

$$
\left\|E_{1}(j) f\right\|_{L^{p}} \leq A(p, j)\|f\|_{L^{p}}
$$

and

$$
\left\|E_{2}(j) f\right\|_{L^{p}} \leq A(p, j)\|f\|_{L^{p}} .
$$

Proof. We give the proof for $E_{1}(j)$. The proof for $E_{2}(j)$ is exactly the same. 
We split $Z_{1}(j)$ as

$$
Z_{1}(j)=\bigcup_{N \geq 0} Z_{1}^{N}(j)
$$

where

$$
Z_{1}^{N}(j)=\left\{(p, q) \in Z_{1}(j):(p, q)=\frac{k+N}{d} \bar{n}_{j-1}+\frac{N}{d} \bar{n}_{j} ; k \in \mathbf{N}\right\} .
$$

(When $j=1$, we add $(0,0)$ to $Z_{1}^{0}(1)$.)

Set

$$
E_{j}^{N}=\sum_{(p, q) \in Z_{1}^{N}(j)}\left(H_{p, q}-S_{p, q}\right)
$$

Then,

$$
E_{1}(j)=\sum_{N \geq 0} E_{j}^{N}
$$

Proposition 4.3 is now a consequence of the following estimate.

5. The $L^{p}$ estimates for $E_{j}^{N}$.

Proposition 5.1. For $1 \leq j \leq r$ and $1<p<\infty$,

$$
\left\|E_{j}^{N} f\right\|_{L^{p}} \leq A(j, p) 2^{-\delta(j, p) N}\|f\|_{L^{p}}
$$

for some $\delta(j, p)>0$.

We first obtain the $L^{2}$ estimate. For that, we need the following improved estimate over estimate (11) for $(p, q) \in Z_{1}^{N}(j)$.

LEMMA 5.2. For $(p, q) \in Z_{1}^{N}(j)$,

$$
\left|m_{p, q}(\xi, \eta, \gamma)\right| \leq A|\gamma| 2^{-\sigma N} 2^{-(p, q) \cdot v_{j}}
$$

for some $\sigma>0$.

Proof. If $(p, q) \in Z_{1}^{N}(j)$, we can write

$$
\begin{aligned}
(p, q) & =\frac{k+N}{d} \bar{n}_{j-1}+\frac{N}{d} \bar{n}_{j} \\
& =\frac{k}{d} \bar{n}_{j-1}+\frac{N}{d}\left(\bar{n}_{j-1}+\bar{n}_{j}\right)
\end{aligned}
$$

for some positive integer $k$. Now it follows from (6) that

$$
\left(v-v_{j}\right) \cdot\left(\bar{n}_{j-1}+\bar{n}_{j}\right)>0 \quad \text { for all } v \in \Lambda \backslash\left\{v_{j}\right\} .
$$

So,

$$
(p, q) \cdot\left(v-v_{j}\right)=\left(\frac{k}{d}\right) \bar{n}_{j-1} \cdot\left(v-v_{j}\right)+\frac{N}{d}\left(\bar{n}_{j-1}+\bar{n}_{j}\right) \cdot\left(v-v_{j}\right) \geq \sigma N
$$

for all $v \in \Lambda \backslash\left\{v_{j}\right\}$ if we choose $\sigma=\frac{1}{d} \min _{v \neq v_{j}}\left\{\left(\bar{n}_{j-1}+\bar{n}_{j}\right) \cdot\left(v-v_{j}\right)\right\}>0$. 
Estimate (13) is now just a consequence of (9), (10), (14) and the mean value theorem.

Proof of Propositon 5.1. It follows from estimate (12) that

$$
\left|m_{p, q}(\xi, \eta, \gamma)\right| \leq \frac{A}{\left(|\gamma| 2^{-(p, q) \cdot v_{j}}\right)^{\epsilon}} .
$$

Taking the convex combination of estimates (13) and (15), we get

$$
\left|m_{p, q}(\xi, \eta, \gamma)\right| \leq C 2^{-\left(\frac{\sigma \epsilon}{1+\epsilon}\right) N} .
$$

Combining estimates (15) and (16), we get

$$
\left|m_{p, q}(\xi, \eta, \gamma)\right| \leq C 2^{-\frac{1}{2}\left(\frac{\sigma \epsilon}{1+\epsilon}\right) N} \frac{1}{\left(|\gamma| 2^{-(p, q) \cdot v_{j}}\right)^{\frac{\epsilon}{2}}} .
$$

Now for $(p, q) \in Z_{1}^{N}(j)$, we have

$$
(p, q)=\frac{k}{d} \bar{n}_{j-1}+\frac{N}{d}\left(\bar{n}_{j-1}+\bar{n}_{j}\right)
$$

for some positive integer $k$. So,

$$
2^{-(p, q) \cdot v_{j}}=2^{-\left(\frac{k}{d}\right)\left(\bar{n}_{j-1} \cdot v_{j}\right)} 2^{-\left(\frac{N}{d}\left(\bar{n}_{j-1}+\bar{n}_{j}\right) \cdot v_{j}\right)}
$$

Let

$$
A_{N}=2^{-\left(\frac{N}{d}\left(\bar{n}_{j-1}+\bar{n}_{j}\right) \cdot v_{j}\right)} \quad \text { and } \quad \delta=\frac{1}{2}\left(\frac{\sigma \epsilon}{1+\epsilon}\right)>0 .
$$

So, estimate (17) is

$$
\left|m_{p, q}(\xi, \eta, \gamma)\right| \leq C 2^{-\delta N} \frac{1}{\left(|\gamma| 2^{-\left(\frac{k}{d}\right)\left(\bar{n}_{j-1} \cdot v_{j}\right)} A_{N}\right)^{\frac{\epsilon}{2}}} .
$$

But $\sigma>\delta$, and so, it follows from (13) and (18) that

$$
\left|m_{p, q}(\xi, \eta, \gamma)\right| \leq C 2^{-\delta N} \min \left(|\gamma| A_{N} 2^{-\frac{k}{d} \bar{n}_{j-1} \cdot v_{j}}, \frac{1}{\left(|\gamma| 2^{-\left(\frac{k}{d}\right)\left(\bar{n}_{j-1} \cdot v_{j}\right)} A_{N}\right)^{\frac{\epsilon}{2}}}\right) .
$$

Since we have assumed in the beginning that no edge of $\mathcal{C}$ passes through the origin, it is easy to see geometrically that $\bar{n}_{j-1} \cdot v_{j} \neq 0$ and so $c_{0}=\left(\bar{n}_{j-1} \cdot v_{j}\right) / d$ is a non-zero constant. Similarly, when dealing with the part $E_{2}(j)$ of $E(j)$, we require $\bar{n}_{j} \cdot v_{j} \neq 0$ that again requires the assumption that $\mathcal{C}$ has no edge through the origin. (We note that this is the first time we have used the fact that no edge of $\mathcal{C}$ passes through the origin). We have

$$
\left|m_{p, q}(\xi, \eta, \gamma)\right| \leq C 2^{-\delta N} \min \left(|\gamma| A_{N} 2^{-k c_{0}}, \frac{1}{\left(|\gamma| A_{N} 2^{-k c_{0}}\right)^{\epsilon / 2}}\right)
$$


So,

$$
\begin{aligned}
& \left|\sum_{(p, q) \in Z_{1}^{N}(j)} m_{p, q}(\xi, \eta, \gamma)\right| \\
& \leq \sum_{(p, q) \in Z_{1}^{N}(j)}\left|m_{p, q}(\xi, \eta, \gamma)\right| \\
& \leq C 2^{-\delta N} \sum_{k:(p, q)=\frac{k}{d} \bar{n}_{j-1}+\frac{N}{d}\left(\bar{n}_{j-1}+\bar{n}_{j}\right)} \min \left(|\gamma| A_{N} 2^{-k c_{0}}, \frac{1}{\left(|\gamma| A_{N} 2^{-k c_{0}}\right)^{\epsilon / 2}}\right) \\
& \leq C 2^{-\delta N} \sum_{k \geq 0} \min \left(|\gamma| A_{N} 2^{-k c_{0}}, \frac{1}{\left(|\gamma| A_{N} 2^{-k c_{0}}\right)^{\epsilon / 2}}\right) \\
& \left.<C 2^{-\delta N}\right)
\end{aligned}
$$

since the sum in $k$ is an absolute constant independent of $\gamma$ and $A_{N}$. Thus, we have shown that the multiplier of $E_{j}^{N}$ is bounded above by $C 2^{-\delta N}$ and so

$$
\left\|E_{j}^{N} f\right\|_{L^{2}} \leq A(j) 2^{-\delta(j) N}\|f\|_{L^{2}}
$$

For other $L^{p}$ estimates, we treat $M_{1}(j)$ and $Q_{1}(j)$ separately. For $(p, q)=\frac{k}{d} \bar{n}_{j-1}+$ $\frac{N}{d}\left(\bar{n}_{j-1}+\bar{n}_{j}\right) \in Z_{1}^{N}(j)$ and $f \in \mathcal{S}$, we have

$$
\begin{aligned}
H_{p, q} f(x, y, z) & =\iint f\left(x-2^{-p} s, y-2^{-q} t, z-2^{-(p, q) \cdot v_{j}} \tilde{P}_{j}(s, t)\right) \psi(s) \psi(t) \mathrm{d} s \mathrm{~d} t \\
& =\iint f\left(x-c_{N} 2^{-\sigma_{1} k} s, y-d_{N} 2^{-\sigma_{2} k} t, z-e_{N} 2^{-\sigma_{3} k} \tilde{P}_{j}(s, t)\right) \psi(s) \psi(t) \mathrm{d} s \mathrm{~d} t
\end{aligned}
$$

where

$$
\begin{array}{rlrl}
c_{N} & =2^{-\frac{N}{d}\left(n_{j-1}^{1}+n_{j}^{1}\right)}, & \sigma_{1} & =\frac{n_{j-1}^{1}}{d} \\
d_{N} & =2^{-\frac{N}{d}\left(n_{j-1}^{2}+n_{j}^{2}\right)}, & \sigma_{2} & =\frac{n_{j-1}^{2}}{d} \\
e_{N}=2^{-\frac{N}{d}\left(\left(\bar{n}_{j-1}+\bar{n}_{j}\right) \cdot v_{j}\right)}, & \sigma_{3} & =\frac{\bar{n}_{j-1} \cdot v_{j}}{d},
\end{array}
$$

recalling that $\bar{n}_{j}=\left(n_{j}^{1}, n_{j}^{2}\right), \bar{n}_{j-1}=\left(n_{j-1}^{1}, n_{j-1}^{2}\right)$ and $v_{j}=\left(m_{j}, n_{j}\right)$.

We note that the polynomial $\tilde{P}_{j}$ also depends on $k, N$ and $j$ since its definition involves $p, q$ and $v_{j}$. Also $c_{N}, d_{N}$ and $e_{N}$ are positive real constants that depend on $N$ and $j$ while $\sigma_{1}, \sigma_{2}$ and $\sigma_{3}$ are arbitrary real constants independent of $N$ but do depend on $j$. We see that $\sigma_{3} \neq 0$ and $\sigma_{1}$ and $\sigma_{2}$ being the components of $\frac{1}{d} \bar{n}_{j-1}$ cannot be zero simultaneously. Moreover, it is clear that if either $\sigma_{1}$ or $\sigma_{2}$ is zero then the other has same sign as $\sigma_{3}$. So without loss of generality, we may assume that $\sigma_{2}$ and $\sigma_{3}$ are of the same sign and that they are positive.

For $k \in \mathbf{N}$ we now define the measures $\mu_{k}^{N,(k)}, v_{k}^{N,(k)}$ and $v^{N,(k)}$ by

$$
<f, \mu_{k}^{N,(k)}>=\iint f\left(c_{N} 2^{-\sigma_{1} k} s, d_{N} 2^{-\sigma_{2} k} t, e_{N} 2^{-\sigma_{3} k} \tilde{P}_{j}(s, t)\right) \psi(s) \psi(t) \mathrm{d} s \mathrm{~d} t
$$




$$
\begin{aligned}
& <f, v_{k}^{N,(k)}>=\iint f\left(2^{-\sigma_{1} k} s, 2^{-\sigma_{2} k} t, 2^{-\sigma_{3} k} \tilde{P}_{j}(s, t)\right) \psi(s) \psi(t) \mathrm{d} s \mathrm{~d} t \\
& <f, v^{N,(k)}>=\iint f\left(s, t, \tilde{P}_{j}(s, t)\right) \psi(s) \psi(t) \mathrm{d} s \mathrm{~d} t
\end{aligned}
$$

for those $k$ 's for which $\frac{k}{d} \bar{n}_{j-1}+\frac{N}{d}\left(\bar{n}_{j-1}+\bar{n}_{j}\right)=(p, q) \in Z_{1}^{N}(j)$ and for all the other $k$ 's, we define them to be zero distribution. We see that $\mu_{k}^{N,(k)}$ is just a fixed dilate of $v_{k}^{N,(k)}$ and also that $v_{k}^{N,(k)}$ 's are the one parameter dilates of $v^{N,(k)}$. So if we set

$$
M_{j}^{N} f=\sum_{(p, q) \in Z_{1}^{N}(j)} H_{p, q} f=\sum_{k>0} \mu_{k}^{N,(k)} * f
$$

and

$$
L_{j}^{N} f=\sum_{k>0} v_{k}^{N,(k)} * f
$$

then it is clear that $\left\|M_{j}^{N}\right\|_{L^{p}-L^{p}}=\left\|L_{j}^{N}\right\|_{L^{p}-L^{p}}$. Thus, it is enough to get the $L^{p}$ estimates for the operator $L_{j}^{N}$. For this, we cannot apply the standard Littlewood-Paley theory because $\sigma_{1}, \sigma_{2}$ and $\sigma_{3}$ may not be of the same sign, and so, here our approach differs from the one in [2]. Our approach shall use Theorems 2.1 and 2.2 that are due to Duoandikoetxea and Rubio de Francia [3]. For convenience, we drop the superscripts $N$ and $(k)$ and denote $v_{k}^{N,(k)}$ by $v_{k}$. We then have the following estimates for $v_{k}$.

LEMMA 5.3.

$$
\left|\widehat{v_{k}}(\xi, \eta, \gamma)\right| \leq C \min \left(2^{-\sigma_{2} k}|\eta|+2^{-\sigma_{3} k}|\gamma|,\left(2^{-\sigma_{2} k}|\eta|+2^{-\sigma_{3} k}|\gamma|\right)^{-1}\right)^{\delta}
$$

for some positive constants $C$ and $\delta$ independent of $N$ and $k$.

Proof. Since

$$
\widehat{v_{k}}(\xi, 0,0)=\iint \exp \left(i \xi 2^{-\sigma_{1} k} s\right) \psi(s) \psi(t) \mathrm{d} s \mathrm{~d} t \equiv 0,
$$

the first (size) estimate for $\widehat{v_{k}}$ follows from the mean value theorem. The second (decay) estimate is just an application of van der Corput's lemma using the fact that $\tilde{P}_{j}(s, t)$ is uniformly in any $C^{m}$ class (for $|s|,|t| \leq 2$ ) and that $\partial^{\alpha} \tilde{P}_{j}$ is uniformly bounded below for some $\alpha$ with $|\alpha| \geq 2$. This completes the proof of Lemma 5.3. We now use these estimates of $\widehat{v_{k}}$ to prove the following important lemma.

LEMMA 5.4. For $1 \leq j \leq r$ and $1<p<\infty$,

$$
\left\|M_{j}^{N} f\right\|_{L^{p}} \leq A(j, p)\|f\|_{L^{p}} .
$$

Proof. Since $\left\|M_{j}^{N} f\right\|_{L^{p}}=\left\|L_{j}^{N} f\right\|_{L^{p}}$, it suffices to show the above estimate for the operator

$$
L_{j}^{N}=\sum_{k>0} v_{k} * f
$$


We shall use Theorem 2.2 to get the $L^{p}$ estimates for $L_{j}^{N}$. We set $\zeta^{0}=\xi, \bar{\zeta}=(\eta, \gamma)$ and

$$
A(t)=\left[\begin{array}{cc}
t^{\sigma_{2}} & 0 \\
0 & t^{\sigma_{3}}
\end{array}\right]
$$

Then, Lemma 5.3 implies that

$$
\left|\widehat{v}_{k}\left(\zeta^{0}, \bar{\zeta}\right)\right| \leq C \min \left(\left|A\left(2^{-k}\right) \bar{\zeta}\right|,\left|A\left(2^{-k}\right) \bar{\zeta}\right|^{-1}\right)^{\delta}
$$

Moreover, $A(s t)=A(s) A(t)$ and $A(t)$ satisfies the Rivière condition since we have assumed that $\sigma_{2}$ and $\sigma_{3}$ are positive. Thus, if we can show that $v^{*}(f)=\sup _{k}|| v_{k}|* f|$ is bounded on $L^{q}\left(\mathbf{R}^{3}\right)$ (with $L^{q}-L^{q}$ norm independent of $N$ ) for all $q>1$, then Lemma 5.4 is just the direct application of Theorem 2.2. We shall apply Theorem 2.1 to $\left\{\left|v_{k}\right|\right\}_{k>0}$ to achieve this where for $f \in \mathcal{S}\left(\mathbf{R}^{3}\right)$,

$$
<f,\left|v_{k}\right|>=\iint f\left(2^{-\sigma_{1} k} s, 2^{-\sigma_{2} k} t, 2^{-\sigma_{3} k} \tilde{P}_{j}(s, t)\right)|\psi(s)||\psi(t)| \mathrm{d} s \mathrm{~d} t .
$$

It is obvious from the definition of $\left|v_{k}\right|$ that the total variation of $\left|v_{k}\right|$ is uniformly (in $k$ and $N$ ) bounded above by $\iint|\psi(s)||\psi(t)| \mathrm{d} s \mathrm{~d} t$. If we follow the proof of estimate (20), then it is easy to see that $\left|v_{k}\right|$ do satisfy estimates (2) and (3) of Theorem 2.1. Now

$$
\left|v_{k}\right|^{(0)} * g(x)=\iint g\left(x-2^{-\sigma_{1} k} s\right)|\psi(s)||\psi(t)| \mathrm{d} s \mathrm{~d} t=C \int g\left(x-2^{-\sigma_{1} k} s\right)|\psi(s)| \mathrm{d} s
$$

and so $v_{(0)}^{*}(g)=\left.\sup _{k}|| v_{k}\right|^{(0)} * g \mid$ is bounded on $L^{q}\left(\mathbf{R}^{1}\right)$ for all $q>1$. But once we have the boundedness for $v_{(0)}^{*}$, the boundedness of $v^{*}$ follows from Theorem 2.1 and this concludes Lemma 5.4.

If we replace $\tilde{P}_{j}(s, t)$ in $\mu_{k}^{N,(k)}$ and $v_{k}^{N,(k)}$ by $a_{m_{j}, n_{j}} s^{m_{j}} t^{n_{j}}$, then using similar techniques, we can show that the operator $Q_{j}^{N}$ defined by $Q_{j}^{N}=\sum_{(p, q) \in Z_{1}^{N}(j)} S_{p, q}$ is bounded on $L^{p}\left(\mathbf{R}^{3}\right), 1<p<\infty$, and also that the norm is independent of $N$. But $E_{j}^{N}=M_{j}^{N}-Q_{j}^{N}$. So,

$$
\left\|E_{j}^{N} f\right\|_{L^{p}} \leq A(j, p)\|f\|_{L^{p}} \quad \text { for } 1<p<\infty .
$$

Proposition 5.1 is now just a consequence of interpolation between the estimates (19) and (21). This completes the proof of Proposition 5.1 and hence Propositions 4.1 and 4.3 also. Thus, $H$ is bounded on $L^{p}\left(\mathbf{R}^{3}\right)$ for $1<p<\infty$ if we prove the following estimate.

\section{The $L^{p}$ estimates for the approximating operators.}

Proposition 6.1. For $1 \leq j \leq r$ and $1<p<\infty$,

$$
\|Q(j) f\|_{L^{p}} \leq A(j, p)\|f\|_{L^{p}}
$$

where $Q(j)=\sum_{(p, q) \in Z(j)} S_{p, q}$ 
Proof. We exploit the Ricci and Stein result to prove the above estimate. For $(p, q) \in Z(j)$,

$$
S_{p, q} f(x, y, z)=\iint f\left(x-2^{-p} s, y-2^{-q} t, z-a_{m_{j}, n_{j}} 2^{-(p, q) \cdot v_{j}} s^{m_{j}} t^{n_{j}}\right) \psi(s) \psi(t) \mathrm{d} s \mathrm{~d} t .
$$

We define

$$
<f, \mu^{(I)}>=\iint f\left(s, t, a_{m_{j}, n_{j}} s^{m_{j}} t^{n_{j}}\right) \psi(s) \psi(t) \mathrm{d} s \mathrm{~d} t
$$

if $I=(p, q)$ is in $Z(j)$ and otherwise $\mu^{(I)}=0$. Thus, the non-zero $\mu^{(I)}$ 's are all identical here. Next we define the dilate $\mu_{I}^{(I)}$ of $\mu^{(I)}$ by

$$
<f, \mu_{I}^{(I)}>=\iint f\left(2^{-p} s, 2^{-q} t, a_{m_{j}, n_{j}} 2^{-(p, q) \cdot v_{j}} s^{m_{j}} t^{n_{j}}\right) \psi(s) \psi(t) \mathrm{d} s \mathrm{~d} t .
$$

Clearly,

$$
Q(j) f=\sum_{I=(p, q) \in Z(j)} S_{p, q} f=\sum_{I=(p, q) \in Z(j)} \mu_{I}^{(I)} * f
$$

It is not hard to see that $\mu^{(I)}$ satisfies all the conditions of Ricci and Stein theorem (Theorem 2.3) and so Proposition 6.1 follows. This also concludes the proof of the sufficiency part of our main theorem in the case when no edge of the convex hull $\mathcal{C}$ passes through the origin.

7. The case of an edge through the origin. We next consider the case when $\mathcal{C}$ has an edge through the origin. It is clear that $\mathcal{C}$ can have a maximum two such edges and also that they cannot be adjacent edges. In this case, the basic idea of the proof is same as before but we need some modifications. We use the same notation as in the previous case.

Once again we show that the operator $H$ is bounded on $L^{p}\left(\mathbf{R}^{3}\right)$ by showing that $M(j)=\sum_{(p, q) \in Z(j)} H_{p, q}$ is bounded on $L^{p}\left(\mathbf{R}^{3}\right)$ for all $1 \leq j \leq r$.

If $v_{j}$ does not lie on the edge through the origin, then we proceed exactly in the same manner as in the previous case. So we assume that one of the $r$ corner points of $\mathcal{C}$, say the corner point $v_{2}=\left(m_{2}, n_{2}\right)$, is on an edge through the origin. Suppose this edge is ${\overline{v_{1}}}_{2}$ and so we have $\bar{n}_{1} \cdot v_{2}=\bar{n}_{1} \cdot v_{1}=0$. (This of course means that $v_{1}$ is also on the edge through the origin and that $\bar{v}_{2} v_{3}$ or $\bar{v}_{r} v_{1}$ cannot be another edge through the origin if it exists). The above proof of $L^{p}$ estimates for $M(j)$ then breaks down for $M(1)$ and $M(2)$ as the proof of Proposition 4.3 requires that $\bar{n}_{j-1} \cdot v_{j}$ and $\bar{n}_{j} \cdot v_{j}$ are non-zero. So we give a separate proof for $M(1)$ and $M(2)$ in this case. In fact, we shall give the proof for $M(2)$ only. The $L^{p}$ estimates of $M(1)$ can be obtained similarly.

We need to show that $M(2)=\sum_{(p, q) \in Z(2)} H_{p, q}$ is bounded on $L^{p}\left(\mathbf{R}^{3}\right)$ for $1<p<\infty$. Set

$$
\mathcal{E}=\left\{v=(m, n) \in \Lambda: v=(m, n) \text { lies on the edge } \bar{v}_{1} v_{2}\right\}
$$

and

$$
P_{\mathcal{E}}(s, t)=\sum_{(m, n) \in \mathcal{E}} a_{m, n} s^{m} t^{n}
$$


For $(p, q) \in Z(2)$ and $f \in \mathcal{S}$, we define

$$
S_{p, q} f(x, y, z)=2^{p+q} \iint f\left(x-s, y-t, z-P_{\mathcal{E}}(s, t)\right) \psi\left(2^{p} s\right) \psi\left(2^{q} t\right) \mathrm{d} s \mathrm{~d} t
$$

and

$$
Q(2)=\sum_{(p, q) \in Z(2)} S_{p, q}
$$

Let $E(2)=M(2)-Q(2)$. Then, we have the following proposition.

Proposition 7.1. For $1<p<\infty$,

$$
\|E(2) f\|_{L^{p}} \leq A(p)\|f\|_{L^{p}} .
$$

Proof. For $(p, q)$ in $Z(2)$, let $m_{p, q}$ denote the multiplier corressponding to $H_{p, q}-$ $S_{p, q}$. Then,

$$
\begin{aligned}
m_{p, q}(\xi, \eta, \gamma)= & \left.\iint \exp \left(i \xi 2^{-p s}+i \eta 2^{-q} t\right)\right)\left[\operatorname { e x p } \left(i \gamma P\left(2^{-p} s, 2^{-q} t\right)\right.\right. \\
& \left.-\exp \left(i \gamma P_{\mathcal{E}}\left(2^{-p} s, 2^{-q} t\right)\right)\right] \psi(s) \psi(t) \mathrm{d} s \mathrm{~d} t
\end{aligned}
$$

We write

$$
\begin{aligned}
P\left(2^{-p} s, 2^{-q} t\right) & =\sum_{v=(m, n) \in \Lambda} a_{m, n} 2^{-p m} 2^{-q n} s^{m} t^{n} \\
& =2^{-(p, q) \cdot v_{2}} \tilde{P}(s, t)
\end{aligned}
$$

where

$$
\tilde{P}(s, t)=\sum_{v=(m, n) \in \Lambda} a_{m, n} 2^{-(p, q) \cdot\left(v-v_{2}\right)} s^{m} t^{n}
$$

and also

$$
P_{\mathcal{E}}\left(2^{-p} s, 2^{-q} t\right)=2^{-(p, q) \cdot v_{2}} \tilde{P}_{\mathcal{E}}(s, t)
$$

where

$$
\tilde{P}_{\mathcal{E}}(s, t)=\sum_{v=(m, n) \in \mathcal{E}} a_{m, n} 2^{-(p, q) \cdot\left(v-v_{2}\right)} s^{m} t^{n}
$$

Thus,

$$
\begin{aligned}
m_{p, q}(\xi, \eta, \gamma)= & \iint \exp \left(i \xi 2^{-p s}+i \eta 2^{-q} t\right)\left[\exp \left(i \gamma 2^{-(p, q) \cdot v_{2}} \tilde{P}(s, t)\right)\right. \\
& \left.-\exp \left(i \gamma 2^{-(p, q) \cdot v_{2}} \tilde{P}_{\mathcal{E}}(s, t)\right)\right] \psi(s) \psi(t) \mathrm{d} s \mathrm{~d} t
\end{aligned}
$$

We then have the following estimate for $m_{p, q}$.

LEMMA 7.2. For $(p, q) \in Z(2)$,

$$
\left|m_{p, q}(\xi, \eta, \gamma)\right| \leq \frac{A}{\left(|\xi| 2^{-p}+|\eta| 2^{-q}+|\gamma| 2^{-(p, q) \cdot v_{2}}\right)^{\epsilon}} \quad \text { for some } \epsilon>0
$$


We omit the proof of this lemma as it is similar to that of Lemma 4.2. The lemma implies that for $(p, q) \in Z(2)$,

$$
\left|m_{p, q}(\xi, \eta, \gamma)\right| \leq \frac{A}{\left(|\gamma| 2^{-(p, q) \cdot v_{2}}\right)^{\epsilon}} .
$$

Next we split

$$
Z(2)=\left\{(p, q) \in \mathbf{Z} \times \mathbf{Z}:(p, q)=\frac{k}{d} \bar{n}_{1}+\frac{l}{d} \bar{n}_{2} ; k \in \mathbf{N}, l \in \mathbf{N}^{\star}\right\}
$$

as $Z(2)=\bigcup_{k \in \mathbf{N}} Z^{k}(2)$ where

$$
Z^{k}(2)=\left\{(p, q) \in Z(2):(p, q)=\frac{k}{d} \bar{n}_{1}+\frac{l}{d} \bar{n}_{2} ; l \in \mathbf{N}^{\star}\right\} .
$$

Let

$$
E_{2}^{k}=\sum_{(p, q) \in Z^{k}(2)} H_{p, q}-S_{p, q}
$$

So,

$$
E(2)=\sum_{k \in \mathbf{N}} E_{2}^{k}
$$

Proposition 7.1 is now a consequence of the following estimate.

Proposition 7.3. For $1<p<\infty$,

$$
\left\|E_{2}^{k}\right\|_{L^{p}} \leq A 2^{-\delta k}\|f\|_{L^{p}} \quad \text { for some } \delta>0 .
$$

The constants $A$ and $\delta$ are independent of $k$.

First, we shall get the $L^{2}$ estimate and for that we need the following estimate.

LEMMA 7.4. For $(p, q) \in Z^{k}(2)$,

$$
\left|m_{p, q}(\xi, \eta, \gamma)\right| \leq A|\gamma| 2^{-\sigma k} 2^{-(p, q) \cdot v_{2}} \quad \text { for some } \sigma>0 .
$$

Proof. We know that

$$
\tilde{P}(s, t)-\tilde{P}_{\mathcal{E}}(s, t)=\sum_{v=(m, n) \in \Lambda \backslash \mathcal{E}} a_{m, n} 2^{-(p, q) \cdot\left(v-v_{2}\right)} s^{m} t^{n} .
$$

Thus if we show that $(p, q) \cdot\left(v-v_{2}\right) \geq \sigma k$ for all $(p, q) \in Z^{k}(2)$ and all $v \in \Lambda \backslash \mathcal{E}$, then estimate (26) is just a consequence of (24) and the mean value theorem. Now

$$
(p, q) \cdot\left(v-v_{2}\right)=\frac{k}{d}\left(\bar{n}_{1} \cdot\left(v-v_{2}\right)\right)+\frac{l}{d}\left(\bar{n}_{2} \cdot\left(v-v_{2}\right)\right) .
$$

But $\bar{n}_{1} \cdot\left(v-v_{2}\right) \geq 0$ and $\bar{n}_{2} \cdot\left(v-v_{2}\right) \geq 0$ for all $v \in \Lambda$ and $\bar{n}_{1} \cdot\left(v-v_{2}\right)=0$ if and only if $v-v_{2}$ is parallel to $\bar{v}_{1} v_{2}$, or in other words, $v$ lies on the edge $\bar{v}_{1} v_{2}$ of $\mathcal{C}$. So for $v \in \Lambda \backslash \mathcal{E}$, we have the following strict inequality:

$$
\bar{n}_{1} \cdot\left(v-v_{2}\right)>0 .
$$


If we now choose

$$
\sigma=\frac{1}{d} \min _{v \in \Lambda \backslash \mathcal{E}}\left\{\bar{n}_{1} \cdot\left(v-v_{2}\right)\right\}>0
$$

then it is easy to see that $(p, q) \cdot\left(v-v_{2}\right) \geq \sigma k$ for all $(p, q) \in Z^{k}(2)$ and for all $v \in \Lambda \backslash \mathcal{E}$.

This completes the proof of Lemma 7.4.

Proof of Proposition 7.3. The rest of the proof for the $L^{2}$ estimate of $E_{2}^{k}$ is now similar to the previous case. Like in the proof of Proposition 5.1, we use the estimates (25) and (26) repeatedly to get

$$
\left|m_{p, q}(\xi, \eta, \gamma)\right| \leq C 2^{-\delta k} \min \left(|\gamma| 2^{-(p, q) \cdot v_{2}}, \frac{1}{\left(|\gamma| 2^{-(p, q) \cdot v_{2}}\right)^{\frac{\epsilon}{2}}}\right)
$$

for some $\delta>0$. But for $(p, q) \in Z^{k}(2)$,

$$
\begin{aligned}
(p, q) \cdot v_{2} & =\frac{k}{d}\left(\bar{n}_{1} \cdot v_{2}\right)+\frac{l}{d}\left(\bar{n}_{2} \cdot v_{2}\right) \\
& =\frac{l}{d}\left(\bar{n}_{2} \cdot v_{2}\right) \quad \text { for some non-negative integer } l .
\end{aligned}
$$

Thus, (27) is the same as

$$
\left|m_{p, q}(\xi, \eta, \gamma)\right| \leq C 2^{-\delta k} \min \left(|\gamma| 2^{-\frac{l}{d}\left(\bar{n}_{2} \cdot v_{2}\right)}, \frac{1}{\left(|\gamma| 2^{-\frac{l}{d}\left(\bar{n}_{2} \cdot v_{2}\right)}\right)^{\frac{\epsilon}{2}}}\right) .
$$

Now $\bar{n}_{2} \cdot v_{2}$ is non-zero since $\bar{n}_{1} \cdot v_{2}=0$. Thus, we can use (28) to sum $m_{p, q}$ 's over all $(p, q) \in Z^{k}(2)$ as in the proof of Proposition 5.1 and this gives

$$
\left|\sum_{(p, q) \in Z^{k}(2)} m_{p, q}(\xi, \eta, \gamma)\right| \leq C 2^{-\delta k}
$$

In other words,

$$
\left\|E_{2}^{k} f\right\|_{L^{2}} \leq A 2^{-\delta k}\|f\|_{L^{2}}
$$

Moreover, using the techniques of Proposition 5.1, we can also deduce that

$$
\left\|E_{2}^{k} f\right\|_{L^{p}} \leq A\|f\|_{L^{p}}, \quad 1<p<\infty .
$$

Proposition 7.3 then follows by interpolating the $L^{p}$ estimate with the $L^{2}$ estimate. To conclude that $M(2)$ is bounded on $L^{p}\left(\mathbf{R}^{3}\right)$, we now just need to prove the following proposition.

Proposition 7.5. For $1<p<\infty$,

$$
\|Q(2) f\|_{L^{p}} \leq A\|f\|_{L^{p}}
$$


where

$$
Q(2)=\sum_{(p, q) \in Z(2)} S_{p, q} .
$$

If we define $R(2)=\sum_{(p, q) \in Z(2)} R_{p, q}$ where

$$
R_{p, q} f(x, y, z)=\iint f\left(x-2^{-p} s, y-2^{-q} t, z-2^{-(p, q) \cdot v_{2}} s^{m_{2}} t^{n_{2}}\right) \psi(s) \psi(t) \mathrm{d} s \mathrm{~d} t
$$

then it follows from the Ricci and Stein result that $R(2)$ is bounded on $L^{p}\left(\mathbf{R}^{3}\right)$. So Proposition 7.5 is a consequence of the following proposition.

Proposition 7.6. For $1<p<\infty$,

$$
\|(Q(2)-R(2)) f\|_{L^{p}} \leq A\|f\|_{L^{p}}
$$

Proof. We recall that

$$
\mathcal{E}=\left\{v=(m, n) \in \Lambda: v=(m, n) \text { lies on the edge } \overline{v_{1} v_{2}} \text { through the origin }\right\} .
$$

Since $\overline{v_{1} v_{2}}$ is an edge through the origin (and $v_{2}$ is the corner point of $\mathcal{C}$ ), it is clear that for all $v=(m, n) \in \mathcal{E}$ either

(i) $m \geq m_{2}$ and $n \geq n_{2}$ or

(ii) $m \leq m_{2}$ and $n \leq n_{2}$.

So without loss of generality, we assume that (i) holds.

Then, for each $v \in \mathcal{E} \backslash\left\{v_{2}\right\}$,

$$
v-v_{2}=\alpha_{v} v_{2}
$$

for some positive real constant $\alpha_{v}$. But we also know that for $(p, q) \in Z(2),(p, q)$. $\left(v-v_{2}\right) \geq 0$ for any $v \in \Lambda$. So in particular for $v \in \mathcal{E} \backslash\left\{v_{2}\right\}$, we have

$$
0 \leq(p, q) \cdot\left(v-v_{2}\right)=(p, q) \cdot \alpha_{v} v_{2}=\alpha_{v}\left((p, q) \cdot v_{2}\right) .
$$

But $\alpha_{v}$ being positive means that $(p, q) \cdot v_{2} \geq 0$ for all $(p, q) \in Z(2)$. In other words, we can write

$$
Z(2)=\bigcup_{N \geq 0} Z^{N}(2)
$$

where

$$
Z^{N}(2)=\left\{(p, q) \in Z(2):(p, q) \cdot v_{2}=p m_{2}+q n_{2}=N\right\} .
$$

This helps to decompose our operator as

$$
\begin{aligned}
Q(2)-R(2) & =\sum_{N \geq 0}\left[Q^{N}(2)-R^{N}(2)\right] \text { where } \\
Q^{N}(2)-R^{N}(2) & =\sum_{(p, q) \in Z^{N}(2)}\left[S_{p, q}-R_{p, q}\right] .
\end{aligned}
$$

Thus, Propostion 7.6 is just a consequence of the following proposition. 
Proposition 7.7. For $1<p<\infty$,

$$
\left\|\left(Q^{N}(2)-R^{N}(2)\right) f\right\|_{L^{p}} \leq A_{p} 2^{-\delta N}\|f\|_{L^{p}}
$$

for some $\delta>0$.

Proof. We first obtain the $L^{2}$ estimate with some decay in $N$. For $(p, q) \in Z^{N}(2)$, let $d_{p, q}$ denote the multiplier of $S_{p, q}-R_{p, q}$. Then for $(p, q) \in Z^{N}(2)$, we have

$$
\begin{aligned}
d_{p, q}(\xi, \eta, \gamma)= & \iint \exp \left(i \xi 2^{-p} s+i \eta 2^{-q} t\right)\left[\exp \left(i \gamma 2^{-N} \tilde{P}_{\mathcal{E}}(s, t)\right)\right. \\
& \left.-\exp \left(i \gamma 2^{-N} a_{m_{2}, n_{2}} s^{m_{2}} t^{n_{2}}\right)\right] \psi(s) \psi(t) \mathrm{d} s \mathrm{~d} t
\end{aligned}
$$

where

$$
\tilde{P}_{\mathcal{E}}(s, t)=a_{m_{2}, n_{2}} s^{m_{2}} t^{n_{2}}+\sum_{v=(m, n) \in \mathcal{E} \backslash\left\{v_{2}\right\}} a_{m, n} 2^{-\alpha_{v} N} s^{m} t^{n} .
$$

If we set $\alpha=\min _{v \in \mathcal{E} \backslash\left\{v_{2}\right\}} \alpha_{v}>0$, then using the mean value theorem and van der Corput's lemma, we deduce that

$$
\left|d_{p, q}(\xi, \eta, \gamma)\right| \leq C \min \left(2^{-\alpha N}\left|\gamma 2^{-N}\right|,\left|\gamma 2^{-N}\right|^{-\epsilon}\right)
$$

which implies that

$$
\left|d_{p, q}(\xi, \eta, \gamma)\right| \leq C 2^{-\theta N}
$$

for some $\theta>0$ (independent of $p$ and $q$ ). We shall now use the additional assumption in the hypothesis of our main theorem. According to this assumption since the edge $\bar{v}_{1} v_{2}$ passes through the origin, no point of $\mathcal{E}$ can have both coordinates odd. If we use this assumption along with the fact that for all $(m, n) \in \mathcal{E}$ the ratio $m / n$ is constant, then at least one of the following must be true:

(i) for all $(m, n) \in \mathcal{E}, m$ is even,

(ii) for all $(m, n) \in \mathcal{E}, n$ is even.

If (i) is true, then $\tilde{P}_{\mathcal{E}}(s, t)$ is an even function of $s$ and if (ii) is true, then $\tilde{P}_{\mathcal{E}}(s, t)$ is an even function of $t$. We assume without loss of generality that $n$ is even for all $(m, n) \in \mathcal{E}$ and so $\tilde{P}_{\mathcal{E}}(s, t)$ is an even function of $t$. Thus,

$$
\iint \exp \left(i \xi 2^{-p} s+i \gamma 2^{-N} \tilde{P}_{\mathcal{E}}(s, t)\right) \psi(s) \psi(t) \mathrm{d} s \mathrm{~d} t \equiv 0
$$

and

$$
\iint \exp \left(i \xi 2^{-p} s+i \gamma 2^{-N} s^{m_{2}} t^{n_{2}}\right) \psi(s) \psi(t) \mathrm{d} s \mathrm{~d} t \equiv 0 .
$$

Using these extra cancellation conditions, it is easy to see that

$$
\left|d_{p, q}(\xi, \eta, \gamma)\right| \leq C\left|\eta 2^{-q}\right|
$$

Using (30), (31) and van der Corput's lemma, we deduce that

$$
\left|d_{p, q}(\xi, \eta, \gamma)\right| \leq C 2^{-\delta N} \min \left(|\eta| 2^{-q},\left(|\eta| 2^{-q}\right)^{-1}\right)^{\epsilon}
$$


for all $(p, q) \in Z^{N}(2)$ and some $\epsilon, \delta>0$. Thus,

$$
\begin{aligned}
\left|\sum_{(p, q) \in Z^{N}(2)} d_{p, q}(\xi, \eta, \gamma)\right| & \leq \sum_{(p, q) \in Z^{N}(2)}\left|d_{p, q}(\xi, \eta, \gamma)\right| \\
& \leq C \sum_{q \in \mathbf{Z}}\left|d_{p, q}(\xi, \eta, \gamma)\right| \text { (assuming } m_{2} \neq 0 \text { for time being) } \\
& \leq C 2^{-\delta N} \sum_{q \in \mathbf{Z}} \min \left(|\eta| 2^{-q},\left(|\eta| 2^{-q}\right)^{-1}\right)^{\epsilon} \\
& \leq C 2^{-\delta N}
\end{aligned}
$$

This implies that

$$
\left\|Q^{N}(2)-R^{N}(2) f\right\|_{L^{2}} \leq C 2^{-\delta N}\|f\|_{L^{2}} .
$$

Using interpolation, Proposition 7.7 now follows from the following proposition.

Proposition 7.8. For $1<p<\infty$,

$$
\left\|\left(Q^{N}(2)-R^{N}(2)\right) f\right\|_{L^{p}} \leq A_{p}\|f\|_{L^{p}} .
$$

Proof. We shall use Theorems 2.1 and 2.2 (due to J. Duoandikoetxea and J. L. Rubio de Francia) to achieve this. We do not need the difference anymore and so we shall prove the above estimate only for $Q^{N}(2)$. The proof for $R^{N}(2)$ is similar. Now

$$
Q^{N}(2) f=\sum_{(p, q) \in Z^{N}(2)} S_{p, q} f=\sum_{q \in \mathbf{Z}} \rho_{q} * f
$$

where we define

$$
<f, \rho_{q}>=\iint f\left(2^{-p} s, 2^{-q} t, 2^{-N} \tilde{P}_{\mathcal{E}}(s, t)\right) \psi(s) \psi(t) \mathrm{d} s \mathrm{~d} t
$$

if ( $\left.p=\frac{N-q n_{2}}{m_{2}}, q\right) \in Z^{N}(2)$ (assuming $m_{2} \neq 0$ for time being) else we define it to be the zero distribution. Then, using the extra cancellation condition

$$
\iint \exp \left(i \xi 2^{-p} s+i \gamma 2^{-N} \tilde{P}_{\mathcal{E}}(s, t)\right) \psi(s) \psi(t) \mathrm{d} s \mathrm{~d} t \equiv 0
$$

and van der Corput's lemma, we get the following estimate for $\widehat{\rho_{q}}$ :

$$
\mid \widehat{\rho}_{q}\left(\xi, \eta, \gamma \mid \leq C \min \left(\left|\eta 2^{-q}\right|,\left|\eta 2^{-q}\right|^{-1}\right)^{\epsilon}\right.
$$

for some $\epsilon>0$. Thus, if we set $\zeta^{0}=(\xi, \gamma)$ and $\bar{\zeta}=\eta$, then Proposition 7.8 follows from Theorem 2.2 once we show that $\rho^{*} f=\sup _{q}|| \rho_{q}|* f|$ is bounded on $L^{\tilde{q}}\left(\mathbf{R}^{3}\right)$ (with $L^{\tilde{q}}-L^{\tilde{q}}$ norm independent of $N$ ) for all $\tilde{q}>1$. But $\left\{\left|\rho_{q}\right|\right\}_{q \in \mathbf{Z}}$ satisfy (2) and (3) (with $\zeta^{0}=(\xi, \gamma), \bar{\zeta}=\eta$ and $\left.A(t)=[t]_{1 \times 1}\right)$ and also $\left\|\rho_{q}\right\| \leq C$ with $C$ uniform in $N$ and $q$. So Theorem 2.1 automatically gives the boundedness of $\rho^{*}$ if we show that $\rho_{(0)}^{*}(g)=$ $\sup _{q} \|\left.\rho_{q}\right|^{(0)} * g \mid$ is bounded on $L^{p}\left(\mathbf{R}^{2}\right)$ for all $p>1$. We shall apply Theorem 2.1 to $\rho_{(0)}^{*}$ 
to achieve this. Now $\rho_{(0)}^{*}(g)=\left.\sup _{q}|| \rho_{q}\right|^{(0)} * g \mid$ and using $p=\frac{N-q n_{2}}{m_{2}}$

$$
<g,\left|\rho_{q}\right|^{(0)}>=\iint g\left(2^{-N / m_{2}} 2^{q n_{2} / m_{2}} s, 2^{-N} \tilde{P}_{\mathcal{E}}(s, t)\right)|\psi(s)||\psi(t)| \mathrm{d} s \mathrm{~d} t .
$$

Now if we define $\omega^{*}(g)=\sup _{q}\left|\omega_{q} * g\right|$ where

$$
\left\langle g, \omega_{q}\right\rangle=\iint g\left(2^{q n_{2} / m_{2}} s, \tilde{P}_{\mathcal{E}}(s, t)\right)|\psi(s)||\psi(t)| \mathrm{d} s \mathrm{~d} t
$$

then it is easy to see that $\left\|\rho_{(0)}^{*}\right\|_{L^{p}-L^{p}}=\left\|\omega^{*}\right\|_{L^{p}-L^{p}}$ for $1<p<\infty$. So without loss of generality, we may assume that

$$
\left\langle g,\left|\rho_{q}\right|^{(0)}\right\rangle=\iint g\left(2^{q n_{2} / m_{2}} s, \tilde{P}_{\mathcal{E}}(s, t)\right)|\psi(s)||\psi(t)| \mathrm{d} s \mathrm{~d} t
$$

and so

$$
\widehat{\left|\rho_{q}\right|^{(0)}}(\xi, \gamma)=\iint \exp \left[i\left(\xi 2^{q n_{2} / m_{2}} s+\gamma \tilde{P}_{\mathcal{E}}(s, t)\right)\right]|\psi(s)||\psi(t)| \mathrm{d} s \mathrm{~d} t .
$$

It is then easy to see that

$$
\left|\widehat{\left|\rho_{q}\right|^{(0)}}(\xi, \gamma)-\widehat{\left|\rho_{q}\right|^{(0)}}(0, \gamma)\right| \leq C\left|2^{q n_{2} / m_{2}} \xi\right|^{\alpha}
$$

and

$$
\left|\widehat{\left|\rho_{q}\right|^{(0)}}(\xi, \gamma)\right| \leq C\left|2^{q n_{2} / m_{2}} \xi\right|^{-\alpha}
$$

for some $\alpha>0$. Thus, $\left\{\left|\rho_{q}\right|^{(0)}\right\}_{q \in \mathbf{Z}}$ satisfy (2) and (3) (with $\zeta^{0}=\gamma, \bar{\zeta}=\xi$ and $A(t)=$ $\left.\left[t^{n_{2} / m_{2}}\right]_{1 \times 1}\right)$ and also $\left\|\rho_{q}^{(0)}\right\| \leq C$ with $C$ uniform in $N$ and $q$. So if we show that $\rho_{(00)}^{*}(h)=$ $\left.\sup _{q}|| \rho_{q}\right|^{(00)} * h \mid$ is bounded on $L^{p}(\mathbf{R})$ for all $p>1$, where

$$
\left\langle h,\left|\rho_{q}\right|^{(00)}\right\rangle=\iint h\left(\tilde{P}_{\mathcal{E}}(s, t)|\psi(s)||\psi(t)| \mathrm{d} s \mathrm{~d} t, \quad h \in \mathcal{S}(\mathbf{R}),\right.
$$

then Theorem 2.1 gives the $L^{p}$ boundedness of $\rho_{(0)}^{*}$ for all $p>1$. But $\left|\rho_{q}\right|^{(00)}$ are all identical (recall that $\tilde{P}_{\mathcal{E}}(s, t)$ only depends on $N$ and not on $q$ ) with $\left\|\rho_{q}^{(00)}\right\| \leq C$ (independent of $N$ ). As a consequence, $\rho_{(00)}^{*}(h)=\left.\sup _{q}|| \rho_{q}\right|^{(00)} * h \mid$ is bounded on $L^{p}(\mathbf{R})$ for all $p>1$ with the operator norm independent of $N$. So by Theorem $2.1, \rho_{(0)}^{*}(g)=$ $\left.\sup _{q}|| \rho_{q}\right|^{(0)} * g \mid$ is bounded on $L^{p}\left(\mathbf{R}^{2}\right)$ for all $p>1$, which in turn implies that $\rho^{*}$ is bounded on $L^{\tilde{q}}\left(\mathbf{R}^{3}\right)$ for all $\tilde{q}>1$. This concludes the proof of Proposition 7.8.

REMARK. We see that the above proofs of Propositions 7.7 and 7.8 work assuming that $m_{2}$ is not zero. However, if it is zero, then $\tilde{P}_{\mathcal{E}}(s, t)$ is a polynomial in only $t$ and for all $(p, q) \in Z^{N}(2), q=N / n_{2}$. We can still apply the same techinques as used in the above proofs but with minor modifications. In fact, in this case using the extra cancellation condition

$$
\iint \exp \left(i \eta 2^{-q} t+i \gamma 2^{-N} \tilde{P}_{\mathcal{E}}(t)\right) \psi(s) \psi(t) \mathrm{d} s \mathrm{~d} t \equiv 0
$$


we can obtain the estimate like (32) in $\xi$ and $p$ and sum $d_{p, q}$ 's in ' $p$ ' rather than in ' $q$ ' as $q=N / n_{2}$ is fixed now. In the proof of Proposition 7.8 also, we sum $Q^{N}(2) f$ in $p$ taking $q$ as $N / n_{2}$ and use similar techniques.

This completes the proof of the sufficiency part of our main theorem apart from the two particular cases.

8. The particular cases. We need to discuss the case when $\mathcal{C}$ consists of just one corner point, (i.e., $P$ is just a monomial) and the case when it consists of two end points say $v_{1}$ and $v_{2}$. It is clear that if $\mathcal{C}$ is just a point, i.e., $P(s, t)$ is a monomial, then the main theorem is just a direct application of the Ricci and Stein theorem. In the latter case, we assume that $\mathcal{C}$ has two edges $\bar{v}_{1} v_{2}$ and $\bar{v}_{2} v_{1}$ (same but considered in opposite directions) and let $\bar{n}_{1}$ and $\bar{n}_{2}\left(=-\bar{n}_{1}\right)$ denote the normals to these edges, respectively.

Now suppose that $\mathcal{C}$ consists of two corner points $v_{1}$ and $v_{2}$ but its edge $\bar{v}_{1} v_{2}$ (or $\bar{v}_{2} v_{1}$ ) does not pass through the origin. The basic structure of the proof in this case is similar to the case where we assumed that $\mathcal{C}$ has at least three corner points. The only difference in this case is that we have to define $Z(1)$ and $Z(2)$ in a slightly different manner. When $\mathcal{C}$ consists of only two corner points $v_{1}$ and $v_{2}, v-v_{1}$ denotes the same direction as $\bar{v}_{1} v_{2}=v_{2}-v_{1}$ for all $v \in \Lambda$. Let $\bar{n}=v-v_{1}$. Then, $v-v_{2}=-\bar{n}$ for all $v \in \Lambda$. We define

$$
T(1)=\left\{(p, q) \in \mathbf{Z} \times \mathbf{Z}:(p, q) \cdot\left(v-v_{1}\right)=(p, q) \cdot \bar{n}>0\right\}
$$

and

$$
T(2)=\left\{(p, q) \in \mathbf{Z} \times \mathbf{Z}:(p, q) \cdot\left(v-v_{2}\right)=(p, q) \cdot(-\bar{n})>0\right\} .
$$

Since $\bar{n}_{1}$ is normal to the edge $\bar{v}_{1} v_{2}=\bar{n}$ and $\bar{n}_{2}\left(=-\bar{n}_{1}\right)$ is normal to the edge $\bar{v}_{2} v_{1}$, it is easy to see that

$$
T(1)=\left\{(p, q) \in \mathbf{Z} \times \mathbf{Z}:(p, q)=\alpha \bar{n}_{1}+\beta \bar{n} ; \alpha \in \mathbf{R}, \beta>0\right\}
$$

and

$$
T(2)=\left\{(p, q) \in \mathbf{Z} \times \mathbf{Z}:(p, q)=\alpha \bar{n}_{2}+\beta(-\bar{n}) ; \alpha \in \mathbf{R}, \beta>0\right\} .
$$

Also from the first definition of $T(1)$ and $T(2)$, it is clear that they are disjoint, and moreover, if we define

$$
Z(1)=T(1) \cup\left\{\alpha \bar{n}_{1} ; \alpha \in \mathbf{R}\right\} \quad \text { and } \quad Z(2)=T(2)
$$

then,

$$
\mathbf{Z} \times \mathbf{Z}=Z(1) \cup Z(2)
$$

Once again we have

$$
\begin{aligned}
Z(1) & =\{(p, q) \in \mathbf{Z} \times \mathbf{Z}:(p, q) \\
& \left.=\frac{k}{d} \bar{n}_{1}+\frac{l}{d} \bar{n} ; k \in \mathbf{Z}, l \in \mathbf{N}^{\star}, \text { where } d \text { is a fixed positive integer }\right\}
\end{aligned}
$$


and

$$
Z(2)=\left\{(p, q) \in \mathbf{Z} \times \mathbf{Z}:(p, q)=\frac{k}{d} \bar{n}_{2}+\frac{l}{d}(-\bar{n}) ; k \in \mathbf{Z} \text { and } l \in \mathbf{N}\right\} .
$$

We now use this decomposition of $\mathbf{Z} \times \mathbf{Z}$ to split our operator $H$ as $H=M(1)+M(2)$ where $M(j)=\sum_{(p, q) \in Z(j)} H_{p, q}$ for $j=1,2$. Thus, it is enough to prove that $M(j)$ is bounded on $L^{p}\left(\mathbf{R}^{3}\right)$ for $j=1,2$ and $1<p<\infty$. Since the basic structure of the proof is same, we discuss it very briefly.

Let $v_{j}=\left(m_{j}, n_{j}\right)$ for $j=1,2$. We define $Q(j)=\sum_{(p, q) \in Z(j)} S_{p, q}$ where

$$
S_{p, q} f(x, y, z)=2^{p+q} \iint f\left(x-s, y-t, z-a_{m_{j}, n_{j}} s^{m_{j}} t^{n_{j}}\right) \psi\left(2^{p} s\right) \psi\left(2^{q} t\right) \mathrm{d} s \mathrm{~d} t .
$$

Let $E(j)=M(j)-Q(j)$. We can prove that $Q(j)$ is bounded on $L^{p}\left(\mathbf{R}^{3}\right)$ by appealing to the Ricci and Stein theorem. So we only need to prove the $L^{p}$ estimates for $E(j)$. For this purpose, we further split our operator $E(j)$ as $E(j)=\sum_{l \geq 0} E_{j}^{l}$ where $E_{j}^{l}=$ $\sum_{(p, q) \in Z^{l}(j)}\left(H_{p, q}-S_{p, q}\right)$ and

$$
Z^{l}(j)=\left\{(p, q) \in Z(j):(p, q)=\frac{k}{d} \bar{n}_{j}+\frac{l}{d}(-1)^{j+1} \bar{n} ; k \in \mathbf{Z}\right\} .
$$

It is then enough to show that

$$
\left\|E_{j}^{l} f\right\|_{L^{p}} \leq A(j, p) 2^{-\delta(j, p) l}\|f\|_{L^{p}} \quad \text { for some } \delta>0 .
$$

We first obtain the above estimate for $p=2$.

For $(p, q) \in Z^{l}(j)$, let $m_{p, q}$ denote the multiplier of $H_{p, q}-S_{p, q}$. Then,

$$
\begin{aligned}
m_{p, q}(\xi, \eta, \gamma)= & \iint \exp \left(i \xi 2^{-p} s+i \eta 2^{-q} t\right)\left[\exp \left(i \gamma P\left(2^{-p} s, 2^{-q} t\right)\right)\right. \\
& \left.-\exp \left(i \gamma 2^{-(p, q) \cdot v_{j}} a_{m_{j}, n_{j}} s^{m_{j}} t^{n_{j}}\right)\right] \psi(s) \psi(t) \mathrm{d} s \mathrm{~d} t .
\end{aligned}
$$

Now for $(p, q) \in Z^{l}(j)$ and $v \in \Lambda \backslash\left\{v_{j}\right\}$, we have

$$
(p, q) \cdot\left(v-v_{j}\right)=(p, q) \cdot\left((-1)^{j+1} \bar{n}\right)>\sigma l
$$

for some $\sigma>0$. (In fact we can choose $\sigma=\frac{|\bar{n}|^{2}}{d}>0$.) Using this fact and van der Corput's lemma, we obtain the following estimates for $m_{p, q}$ :

$$
\left|m_{p, q}(\xi, \eta, \gamma)\right| \leq A|\gamma| 2^{-\sigma l} 2^{-(p, q) \cdot v_{j}}
$$

and

$$
\left|m_{p, q}(\xi, \eta, \gamma)\right| \leq A\left(|\gamma| 2^{-(p, q) \cdot v_{j}}\right)^{-\epsilon} \quad \text { for some } \epsilon>0
$$

Using these estimates, we sum $m_{p, q}$ 's over all $(p, q) \in Z^{l}(j)$ and get

$$
\left\|E_{j}^{l} f\right\|_{L^{2}} \leq A(j) 2^{-\delta(j) l}\|f\|_{L^{2}} \quad \text { for } j=1,2 .
$$


Also, using the previous techniques and the theorems due to J. Duoandikoetxea an J. L. Rubio de Francia, we can get the estimate

$$
\left\|E_{j}^{l} f\right\|_{L^{p}} \leq A(j, p)\|f\|_{L^{p}} \text { for } 1<p<\infty .
$$

Estimate (35) now follows from the estimates (36) and (37) and so we are done in this particular case.

Now suppose $\mathcal{C}$ consists of two corner points $v_{1}$ and $v_{2}$ and its edge passes through the origin. In this case, as we saw earlier (because of the assumption that no point of $\Lambda$ has both coordinates odd on this edge) that the polynomial $P$ will be an even function of either $s$ or $t$. We split the region of integration into $Z(1)$ and $Z(2)$ as defined in (33) and (34). Now for $j=1,2$, define $Q(j)=\sum_{(p, q) \in Z(j)} S_{p, q}$ where

$$
S_{p, q} f(x, y, z)=\iint f\left(x-2^{-p} s, y-2^{-q} t, z-2^{-(p, q) \cdot v_{j}} \tilde{P}_{j}(s, t)\right) \psi(s) \psi(t) \mathrm{d} s \mathrm{~d} t
$$

and $\tilde{P}_{j}(s, t)=2^{(p, q) \cdot v_{j}} P\left(2^{-p} s, 2^{-q} t\right)$. Once again, it can be verified that $Q(j)$ is bounded on $L^{p}\left(\mathbf{R}^{3}\right)$ for $j=1,2$ and $1<p<\infty$. This can be done exactly in the same manner as in Proposition 7.5. But in this case,

$$
H=\sum_{(p, q) \in \mathbf{Z} \times \mathbf{Z}} S_{p, q}=Q(1)+Q(2) .
$$

Thus, $Q(1)$ and $Q(2)$ bounded implies that $H$ is bounded on $L^{p}\left(\mathbf{R}^{3}\right)$ for $1<p<\infty$ and so we are done in this particular case also.

This completes the proof of the sufficiency part of our main theorem in all the possible cases. We next prove the necessary part of the theorem.

9. The proof of the necessary part of the main theorem. First we show that if any of the corner point of the convex hull $\mathcal{C}$ has both coordinates odd, then $H$ is not bounded on $L^{2}$. Suppose $(k, l)$ is a corner point of $\mathcal{C}$ with both $k$ and $l$ odd. Without loss of generality, we assume that the coefficient $a_{k, l}=1$. Since $(k, l)$ is a corner point, there are numbers $a$ and $b$ so that $a k+b l<a m+b n$ for every $(m, n)$ in $\Lambda \backslash\{(k, l)\}$.

Now $H f=\mu * f$, where $\mu$ is a tempered distribution defined by

$$
\langle\phi, \mu\rangle=p . v . \iint \phi(s, t, P(s, t)) \frac{\mathrm{d} s \mathrm{~d} t}{s t}, \quad \phi \in \mathcal{S} .
$$

We define the dilate $\mu_{\delta}$ of $\mu$ by $\mu_{\delta}(\phi)=\mu\left(\phi_{\delta}\right)$, where

$$
\phi_{\delta}(s, t, u)=\phi\left(\delta^{-a} s, \delta^{-b} t, \delta^{-(a k+b l)} u\right) .
$$

We then show that $\mu_{\delta} \rightarrow v$ (in the sense of distributions) as $\delta \rightarrow 0$, where

$$
\langle\phi, v\rangle=p . v . \iint \phi\left(s, t, s^{k} t^{l}\right) \frac{\mathrm{d} s \mathrm{~d} t}{s t} .
$$

Now if $f \mapsto \mu * f$ were bounded on $L^{2}\left(\mathbf{R}^{3}\right)$, then $f \mapsto \mu_{\delta} * f$ would be uniformly bounded on $L^{2}\left(\mathbf{R}^{3}\right)$, and so $f \mapsto v * f$ would be bounded on $L^{2}\left(\mathbf{R}^{3}\right)$. But we shall see (Section 9.1), $f \mapsto v * f$ is not bounded on $L^{2}\left(\mathbf{R}^{3}\right)$. Thus to prove our claim, we just need to show that $\mu_{\delta} \rightarrow v$ as $\delta \rightarrow 0$. But this is shown in [2]. 
Finally to conclude Theorem 1.2, we show that if any point of $\Lambda$ on an edge of $\mathcal{C}$ through the origin has both coordinates odd, then $H$ is not bounded on $L^{2}$. To show this of course, we can now assume that all the corner points of $\mathcal{C}$ have at least one even coordinate otherwise the above proof works. Suppose the edge through the origin is $\bar{v}_{1} v_{2}$. Then, $v_{1}$ and $v_{2}$ being the corner points have at least one even coordinate. We again set

$$
\mathcal{E}=\left\{v=(m, n) \in \Lambda: v=(m, n) \text { lies on the edge }{\overline{v_{1}}}_{2}\right\}
$$

and

$$
P_{\mathcal{E}}(s, t)=\sum_{(m, n) \in \mathcal{E}} a_{m, n} s^{m} t^{n} .
$$

Now suppose for some $v=(m, n) \in \mathcal{E}$, both $m$ and $n$ are odd. Since all the points of $\mathcal{E}$ lie on the edge $\bar{v}_{1} v_{2}$, there exist numbers $a$ and $b$ such that $a m+b n=0$ whenever $(m, n) \in \mathcal{E}$ and $a m+b n>0$ whenever $(m, n) \in \Lambda \backslash \mathcal{E}$. We define the dilate $\mu_{\delta}$ of $\mu$ by $\mu_{\delta}(\phi)=\mu\left(\phi_{\delta}\right)$, where

$$
\phi_{\delta}(s, t, u)=\phi\left(\delta^{-a} s, \delta^{-b} t, u\right)
$$

We then show that $\mu_{\delta} \rightarrow \sigma$ as $\delta \rightarrow 0$, where

$$
\langle\phi, \sigma\rangle=p . v \cdot \iint \phi\left(s, t, P_{\mathcal{E}}(s, t)\right) \frac{\mathrm{d} s \mathrm{~d} t}{s t} .
$$

But $f \mapsto \sigma * f$ is not bounded on $L^{2}\left(\mathbf{R}^{3}\right)$ (see below) and so to conclude that $H$ is not bounded on $L^{2}\left(\mathbf{R}^{3}\right)$, it is enough to show that $\mu_{\delta} \rightarrow \sigma$ as $\delta \rightarrow 0$.

After a change of variables, we see that

$$
\left\langle\phi, \mu_{\delta}\right\rangle=\iint \phi\left(s, t, P_{\delta}(s, t)\right) \frac{\mathrm{d} s \mathrm{~d} t}{s t}
$$

where $P_{\delta}(s, t)=P_{\mathcal{E}}(s, t)+\sum_{(m, n) \in \Lambda \backslash \mathcal{E}} \delta_{m, n} a_{m, n} s^{m} t^{n}$ with $\epsilon_{m, n}=a m+b n>0$ for $(m, n) \in$ $\Lambda \backslash \mathcal{E}$. If $\alpha$ and $\beta$ are fixed small positive numbers, then

$$
\begin{aligned}
\left\langle\phi, \mu_{\delta}-\sigma\right\rangle= & \int_{|s| \leq 1 / \delta^{\alpha}} \int_{|t| \leq 1 / \delta^{\beta}}\left[\phi\left(s, t, P_{\delta}(s, t)\right)-\phi\left(s, t, P_{\mathcal{E}}(s, t)\right)\right] \frac{\mathrm{d} s \mathrm{~d} t}{s t} \\
& +\int_{\substack{|s| \geq 1 / \delta^{\alpha}\\
}} \phi\left(s, t, P_{\delta}(s, t)\right) \frac{\mathrm{d} s \mathrm{~d} t}{s t}+\int_{\substack{|s| \leq 1 / \delta^{\alpha} \\
|t| \geq 1 / \delta^{\beta}}} \phi\left(s, t, P_{\delta}(s, t)\right) \frac{\mathrm{d} s \mathrm{~d} t}{s t} \\
& -\int_{\substack{|s| \leq 1 / \delta^{\alpha} \\
|t| \geq 1 / \delta^{\beta}}} \phi\left(s, t, P_{\mathcal{E}}(s, t)\right) \frac{\mathrm{d} s \mathrm{~d} t}{s t}-\int_{\substack{|s| \geq 1 / \delta^{\alpha} \\
|t| \leq 1 / \delta^{\beta}}} \phi\left(s, t, P_{\mathcal{E}}(s, t)\right) \frac{\mathrm{d} s \mathrm{~d} t}{s t} \\
& -\int_{\substack{|s| \geq 1 / \delta^{\alpha} \\
|t| \geq 1 / \delta^{\beta}}} \phi\left(s, t, P_{\mathcal{E}}(s, t)\right) \frac{\mathrm{d} s \mathrm{~d} t}{s t} \\
:= & A(\delta)+B(\delta)+C(\delta)-D(\delta)-E(\delta)-F(\delta) .
\end{aligned}
$$


We can show that $A(\delta), B(\delta), C(\delta)$ and $F(\delta)$ tend to zero as $\delta \rightarrow 0$, as in [2]. So we give the proof for $D(\delta)$ and $E(\delta)$ only. We write

$$
\begin{aligned}
D(\delta)= & \int_{\substack{|s| \leq 1 / \delta^{\alpha} \\
|t| \geq 1 / \delta^{\beta}}}\left[\phi\left(s, t, P_{\mathcal{E}}(s, t)\right)-\phi(s, t, 0)\right] \frac{\mathrm{d} s \mathrm{~d} t}{s t} \\
& +\int_{\substack{|s| \leq 1 / \delta^{\alpha} \\
|t| 1 / \delta^{\beta}}}[\phi(s, t, 0)-\phi(0, t, 0)] \frac{\mathrm{d} s \mathrm{~d} t}{s t} \\
:= & G(\delta)+H(\delta) .
\end{aligned}
$$

It is now easy to see that $H(\delta) \rightarrow 0$ as $\delta \rightarrow 0$. We write $G(\delta)=G_{1}(\delta)+G_{2}(\delta)$ where the integrands in $G_{1}$ and $G_{2}$ are same but the region of integration in $G_{1}$ is $|s| \leq 1,|t| \geq$ $(1 / \delta)^{\beta}$ and for $G_{2} 1 \leq|s| \leq(1 / \delta)^{\alpha},|t| \geq(1 / \delta)^{\beta}$. It is once again easy to see that

$$
\left|G_{1}(\delta)\right| \leq C \int_{\substack{|s| \leq 1 \\|t| \geq(1 / \delta)^{\beta}}} \frac{|s|^{u / 2}|t|^{w / 2}}{|t|^{N / 2}} \frac{\mathrm{d} s \mathrm{~d} t}{|s||t|}
$$

where $u=\min \{m:(m, n) \in \mathcal{E}\} \geq 1, w=\max \{n:(m, n) \in \Lambda\}$ and $N$ is as big as we want. This implies that $G_{1}(\delta)=\mathcal{O}\left(\delta^{\beta / 2(N-w)}\right)$ and so $G_{1}(\delta) \rightarrow 0$ as $\delta \rightarrow 0$ if $N$ is large enough. The same can be verified for $G_{2}(\delta)$. Thus, $D(\delta) \rightarrow 0$ as $\delta \rightarrow 0$. Interchanging the role of $s$ and $t$, we can prove the same thing for $E(\delta)$. Summing up, $\mu_{\delta}(\phi) \rightarrow \sigma(\phi)$ as $\delta \rightarrow 0$. This concludes that $H$ is not bounded on $L^{2}\left(\mathbf{R}^{3}\right)$ in this case also.

Finally, we give the proof of the fact that $f \mapsto \sigma * f$ is not bounded on $L^{2}\left(\mathbf{R}^{3}\right)$.

9.1. Proof that $f \mapsto v * f$ and $f \mapsto \sigma * f$ not bounded on $L^{2}\left(\mathbf{R}^{3}\right)$. It suffices to show that $\widehat{\nu_{\epsilon, \epsilon^{\prime}, R, R^{\prime}}}(\xi, \eta, \gamma)$ and $\widehat{\sigma_{\epsilon, \epsilon^{\prime}, R, R^{\prime}}}(\xi, \eta, \gamma)$ are not uniformly (in $\epsilon, \epsilon^{\prime}, R$ and $R^{\prime}$ ) bounded functions almost everywhere, where

$$
\widehat{v_{\epsilon, \epsilon^{\prime}, R, R^{\prime}}}(\xi, \eta, \gamma)=\int_{\epsilon^{\prime} \leq|s| \leq R^{\prime}} \int_{\epsilon \leq|t| \leq R} \exp \left(i\left(\xi s+\eta t+\gamma s^{k} t^{l}\right)\right) \frac{\mathrm{d} s \mathrm{~d} t}{s t}
$$

and

$$
\widehat{\sigma_{\epsilon, \epsilon^{\prime}, R, R^{\prime}}}(\xi, \eta, \gamma)=\int_{\epsilon^{\prime} \leq|s| \leq R^{\prime}} \int_{\epsilon \leq|t| \leq R} \exp \left[i\left(\xi s+\eta t+\gamma P_{\mathcal{E}}(s, t)\right)\right] \frac{\mathrm{d} s \mathrm{~d} t}{s t}
$$

But being continuous functions, it furthermore suffices to show that $\widehat{v_{\epsilon, \epsilon^{\prime}, R, R^{\prime}}}(0,0, \gamma)$ and $\widehat{\sigma_{\epsilon, \epsilon^{\prime}, R, R^{\prime}}}(0,0, \gamma)$ are not uniformly bounded functions. Now

$$
\begin{aligned}
\widehat{v_{\epsilon, \epsilon^{\prime}, R, R^{\prime}}}(0,0, \gamma) & =\int_{\epsilon^{\prime} \leq|s| \leq R^{\prime}} \frac{\mathrm{d} s}{s} \int_{\epsilon \leq|t| \leq R} \exp \left(i \gamma s^{k} t^{l}\right) \frac{\mathrm{d} t}{t} \\
& =2 \int_{\epsilon^{\prime} \leq s \leq R^{\prime}} \frac{\mathrm{d} s}{s} \int_{\epsilon \leq|t| \leq R} \exp \left(i \gamma s^{k} t^{l}\right) \frac{\mathrm{d} t}{t} \\
& =2 \int_{\epsilon^{\prime} \leq s \leq R^{\prime}} \frac{\mathrm{d} s}{s} \frac{1}{l} \int_{s^{k} \epsilon^{l} \leq|u| \leq s^{k} R^{l}} \exp (i \gamma u) \frac{\mathrm{d} u}{u} \\
& =\frac{4 i}{l} \int_{\epsilon^{\prime} \leq s \leq R^{\prime}} \frac{\mathrm{d} s}{s} \int_{s^{k} \epsilon^{l} \leq u \leq s^{k} R^{l}} \sin (\gamma u) \frac{\mathrm{d} u}{u} .
\end{aligned}
$$


Then,

$$
\lim _{\substack{\epsilon \rightarrow 0 \\ R \rightarrow \infty}} \widehat{\epsilon_{\epsilon, \epsilon^{\prime}, R, R^{\prime}}}(0,0, \gamma)=\frac{4 \pi i}{2 l} \log \frac{R^{\prime}}{\epsilon^{\prime}}
$$

and so $\widehat{v_{\epsilon, \epsilon^{\prime}, R, R^{\prime}}}$ is not an uniformly bounded function in $\epsilon^{\prime}$ and $R^{\prime}$. We now show that

$$
\begin{aligned}
\widehat{\sigma_{\epsilon, \epsilon^{\prime}, R, R^{\prime}}}(0,0, \gamma) & =\int_{\epsilon^{\prime} \leq|s| \leq R^{\prime}} \frac{\mathrm{d} s}{s} \int_{\epsilon \leq|t| \leq R} \exp \left(i \gamma P_{\mathcal{E}}(s, t)\right) \frac{\mathrm{d} t}{t} \\
& =\int_{\epsilon^{\prime} \leq|s| \leq R^{\prime}} \frac{\mathrm{d} s}{s} \int_{\epsilon \leq|t| \leq R} \exp \left(i \gamma \sum_{(m, n) \in \mathcal{E}} a_{m, n} s^{m} t^{n}\right) \frac{\mathrm{d} t}{t}
\end{aligned}
$$

is not uniformly bounded. We recall that

$$
\mathcal{E}=\left\{v=(m, n) \in \Lambda: v=(m, n) \text { lies on the edge } \bar{v}_{1} v_{2} \text { through the origin }\right\}
$$

and so for all $(m, n) \in \mathcal{E}$, the ratio $m / n$ is the same. Also we have assumed that for some $(m, n) \in \mathcal{E}$, both $m$ and $n$ are odd. These two facts allow us to conclude that for any $(m, n) \in \mathcal{E}$, either both $m$ and $n$ are odd or both are even. In particular, both the coordinates of $v_{1}$ and $v_{2}$ have to be even. It is also clear that there exist odd (positive) integers $p$ and $q$ that are relatively prime and such that

$$
m=\frac{p}{q} n \quad \text { for all }(m, n) \in \mathcal{E} .
$$

Also $q$ being relatively prime to $p$, must divide $n$ and so $n / q$ is always a positive integer. Furthermore, it is even if $n$ is even and is odd if $n$ is odd. Thus, if $v_{1}=\left(m_{1}, n_{1}\right)$ and $v_{2}=\left(m_{2}, n_{2}\right)$, then $n_{1} / q$ and $n_{2} / q$ are both even. The change of variable $s^{p} t^{q}=u$ in the $t$ integral of $\widehat{\sigma_{\epsilon, \epsilon^{\prime}, R, R^{\prime}}}(0,0, \gamma)$ then gives

$$
\widehat{\sigma_{\epsilon, \epsilon^{\prime}, R, R^{\prime}}}(0,0, \gamma)=2 \int_{\epsilon^{\prime} \leq s \leq R^{\prime}} \frac{\mathrm{d} s}{s} \frac{1}{q} \int_{s^{p} \epsilon^{q} \leq|u| \leq s^{p} R^{q}} \exp \left(i \gamma \sum_{(m, n) \in \mathcal{E}} a_{m, n} u^{n / q}\right) \frac{\mathrm{d} u}{u}
$$

Therefore,

$$
\lim _{\substack{\epsilon \rightarrow 0 \\ R \rightarrow \infty}} \widehat{\sigma_{\epsilon, \epsilon^{\prime}, R, R^{\prime}}}(0,0, \gamma)=\frac{2}{q} M(\gamma) \log \frac{R^{\prime}}{\epsilon^{\prime}}
$$

where

$$
M(\gamma)=\int_{-\infty}^{\infty} \exp (i \gamma P(u)) \frac{\mathrm{d} u}{u}
$$

and $P(u)=\sum_{(m, n) \in \mathcal{E}} a_{m, n} u^{n / q}$ is some polynomial in $u$ such that the highest and the lowest power of $u$ in $P(u)$ is even and also it has at least one monomial with odd power. But as we shall see, $M(\gamma) \not \equiv 0$ and so it follows that $\widehat{\sigma_{\epsilon, \epsilon^{\prime}, R, R^{\prime}}}$ is not uniformly bounded in $\epsilon^{\prime}$ and $R^{\prime}$. This completes the proof of the necessity part of the main theorem. Thus to conclude the proof of the main theorem, we just need to show that $M(\gamma) \not \equiv 0$. We show this by developing the asymptotic expansion of $M(\gamma)$. 
9.2. Asymptotic expansion of $M(\gamma)$. We show that

$$
M(\gamma)=\int_{-\infty}^{\infty} \exp (i \gamma P(v)) \frac{d v}{v} \not \equiv 0
$$

where $P(v)$ is some polynomial in $v$ such that the highest and the lowest power of $v$ in $P(v)$ is even and also it has at least one monomial with odd power. We shall assume without loss of generality that all the coefficients of $P(v)$ are equal to 1 . It can be verified that the proof below works in all other cases. From the definition of $P(v)$, it is clear that the integral $M(\gamma)$ can be written as

$$
M(\gamma)=2 i \int_{0}^{\infty} e^{i \gamma\left(v^{\alpha_{1}}+v^{\alpha_{2}}+\cdots+v^{\alpha_{S}}\right)} \sin \gamma\left(v^{\beta_{1}}+v^{\beta_{2}}+\cdots+v^{\beta_{t}}\right) \frac{d v}{v}
$$

where $\alpha_{i}$ 's are all positive even integers, $\beta_{j}$ 's are all positive odd integers with $\alpha_{1}<\beta_{1}$, $\alpha_{s}>\beta_{t}, \alpha_{i}<\alpha_{j}$ for $1 \leq i<j \leq s$ and $\beta_{i}<\beta_{j}$ for $1 \leq i<j \leq t$.

The factor $2 i$ in the above integral of $M(\gamma)$ is not of much importance and so we shall ignore it from now.

(In what follows, ' $c$ ' denotes a general constant that depends on the $\alpha_{i}$ 's and $\beta_{j}$ 's but not on $\gamma$ ).

If we now make a change of variable $\gamma^{1 / \alpha_{s}} v=u$, then

$$
M(\gamma)=\int_{0}^{\infty} e^{i\left(\gamma^{1-\frac{\alpha_{1}}{\alpha_{s}}} u^{\alpha_{1}}+\gamma^{1-\frac{\alpha_{2}}{\alpha_{s}}} u^{\alpha_{2}}+\cdots+u^{\alpha_{s}}\right)} \sin \left(\gamma^{1-\frac{\beta_{1}}{\alpha_{s}}} u^{\beta_{1}}+\gamma^{1-\frac{\beta_{2}}{\alpha_{s}}} u^{\beta_{2}}+\cdots+\gamma^{1-\frac{\beta_{t}}{\alpha_{s}}} u^{\beta_{t}}\right) \frac{d u}{u} .
$$

Now let

$$
\phi(u)=\gamma^{1-\frac{\alpha_{1}}{\alpha_{s}}} u^{\alpha_{1}}+\gamma^{1-\frac{\alpha_{2}}{\alpha_{s}}} u^{\alpha_{2}}+\cdots+u^{\alpha_{s}}
$$

and

$$
\psi(u)=\gamma^{1-\frac{\beta_{1}}{\alpha_{s}}} u^{\beta_{1}}+\gamma^{1-\frac{\beta_{2}}{\alpha_{s}}} u^{\beta_{2}}+\cdots+\gamma^{1-\frac{\beta_{t}}{\alpha_{s}}} u^{\beta_{t}} .
$$

Then,

$$
\begin{aligned}
M(\gamma) & =\int_{0}^{1 / \gamma^{\theta}} e^{i \phi(u)} \sin (\psi(u)) \frac{d u}{u}+\int_{1 / \gamma^{\theta}}^{\infty} e^{i \phi(u)} \sin (\psi(u)) \frac{d u}{u} \\
& :=A(\gamma)+B(\gamma)
\end{aligned}
$$

where we choose $\theta$ such that

$$
0<\frac{\alpha_{s}-\beta_{t}}{\alpha_{s}^{2}}<\theta<\frac{\alpha_{s}-\beta_{t}}{\alpha_{s} \beta_{t}}<\frac{\alpha_{s}-\beta_{t}}{\alpha_{s}}=k<1 .
$$

We claim that for $\gamma$ sufficiently small,

$$
M(\gamma)=c \gamma^{k}+O\left(\gamma^{l}\right) \quad \text { with } c \text { a non-zero constant and } l>k .
$$


In other words, $c \gamma^{k}$ is the principal term in the asymptotic expansion of $M(\gamma)$ when $\gamma$ is small enough. It then follows that

$$
M(\gamma) \not \equiv 0
$$

We first show that $B(\gamma)$ is an error term, i.e., $B(\gamma)=O\left(\gamma^{l}\right)$ for $l>k$. Clearly,

$$
B(\gamma)=\frac{1}{2 i} \int_{1 / \gamma^{\theta}}^{\infty} e^{i(\phi(u)+\psi(u))} \frac{d u}{u}-\frac{1}{2 i} \int_{1 / \gamma^{\theta}}^{\infty} e^{i(\phi(u)-\psi(u))} \frac{d u}{u} .
$$

If $\gamma$ is sufficiently small and $\tau(u)=\phi(u)+\psi(u)$, we see that for $u \geq 1 / \gamma^{\theta}$,

$$
\left|\tau^{\prime}(u)\right| \geq c u^{\alpha_{s}-1} \quad \text { and } \quad\left|\tau^{\prime \prime}(u)\right| \leq c u^{\alpha_{s}-2} .
$$

Now if we integrate by parts and use the above estimates of $\tau^{\prime}$ and $\tau^{\prime \prime}$, then it is clear that $B(\gamma)=O\left(\gamma^{\alpha_{s} \theta}\right)$. But $\alpha_{s} \theta>k$ and so $B(\gamma)$ is an error term. Also

$$
\begin{aligned}
A(\gamma) & =\int_{0}^{1 / \gamma^{\theta}}\left(e^{i \phi(u)}-e^{i u^{\alpha_{s}}}\right) \sin \psi(u) \frac{d u}{u}+\int_{0}^{1 / \gamma^{\theta}} e^{i u^{\alpha_{s}}} \sin \psi(u) \frac{d u}{u} \\
& :=C(\gamma)+D(\gamma) .
\end{aligned}
$$

But

$$
\begin{aligned}
C(\gamma) & =\int_{0}^{\gamma^{1 / \alpha_{s}}}\left(e^{i \phi(u)}-e^{i u^{\alpha_{s}}}\right) \sin \psi(u) \frac{d u}{u}+\int_{\gamma^{1 / \alpha_{s}}}^{1 / \gamma^{\theta}}\left(e^{i \phi(u)}-e^{i u^{\alpha_{s}}}\right) \sin \psi(u) \frac{d u}{u} \\
& :=E(\gamma)+F(\gamma)
\end{aligned}
$$

and

$$
|E(\gamma)| \leq \gamma^{1-\frac{\alpha_{1}}{\alpha_{s}}} \int_{0}^{\gamma^{1 / \alpha_{s}}} u^{\alpha_{1}} \frac{d u}{u}+\cdots+\gamma^{1-\frac{\alpha_{s-1}}{\alpha_{s}}} \int_{0}^{\gamma^{1 / \alpha_{s}}} u^{\alpha_{s-1}} \frac{d u}{u}=O(\gamma) .
$$

So $E(\gamma)$ is also an error term. We now consider $F(\gamma)$.

Since $\sin \psi(u)=O(\psi(u))$, we have

$$
\sin \psi(u)=O\left(\gamma^{1-\frac{\beta_{1}}{\alpha_{s}}} u^{\beta_{1}}\right) \quad \text { for } u \leq \gamma^{1 / \alpha_{s}}
$$

and

$$
\sin \psi(u)=O\left(\gamma^{1-\frac{\beta_{t}}{\alpha_{s}}} u^{\beta_{t}}\right) \quad \text { for } u \geq \gamma^{1 / \alpha_{s}} .
$$

Let $\theta_{1}=\frac{1}{\alpha_{s}}\left(\frac{\alpha_{s}-\alpha_{s-1}}{\alpha_{s-1}+\beta_{t}}\right)$. Using (39), we have

$$
\begin{aligned}
|F(\gamma)| & \leq c \gamma^{1-\frac{\beta_{t}}{\alpha_{s}}}\left(\gamma^{1-\frac{\alpha_{1}}{\alpha_{s}}} \int_{\gamma^{1} / \alpha_{s}}^{1 / \gamma^{\theta}} u^{\alpha_{1}+\beta_{t}} \frac{d u}{u}+\cdots \gamma^{1-\frac{\alpha_{s-1}}{\alpha_{s}}} \int_{\gamma^{1} / \alpha_{s}}^{1 / \gamma^{\theta}} u^{\alpha_{s-1}+\beta_{t}} \frac{d u}{u}\right) \\
& \leq c \gamma^{1-\frac{\beta_{t}}{\alpha_{s}}+1-\frac{\alpha_{s-1}}{\alpha_{s}}}\left[\left(\gamma^{-\theta\left(\alpha_{1}+\beta_{t}\right)}+\gamma^{\frac{\alpha_{1}+\beta_{t}}{\alpha_{s}}}\right)+\cdots\left(\gamma^{-\theta\left(\alpha_{s-1}+\beta_{t}\right)}+\gamma^{\frac{\alpha_{s-1}+\beta_{t}}{\alpha_{s}}}\right)\right] \\
& \leq c \gamma^{1-\frac{\beta_{t}}{\alpha_{s}}+1-\frac{\alpha_{s-1}}{\alpha_{s}}-\theta\left(\alpha_{s-1}+\beta_{t}\right)} .
\end{aligned}
$$


But

$$
1-\frac{\beta_{t}}{\alpha_{s}}=k
$$

and so if we show that

$$
1-\frac{\alpha_{s-1}}{\alpha_{s}}-\theta\left(\alpha_{s-1}+\beta_{t}\right)>0
$$

then $F(\gamma)$ is also an error term. But this is same as showing

$$
\frac{\alpha_{s}-\alpha_{s-1}}{\alpha_{s}}>\theta\left(\alpha_{s-1}+\beta_{t}\right)
$$

which holds if $\theta_{1}>\theta$. However, if $\theta_{1}<\theta$, we choose $\theta_{2}$ such that $0<\theta_{2}<\theta_{1}$ and write $F(\gamma)$ as

$$
\begin{aligned}
F(\gamma)= & \int_{\gamma^{1 / \alpha_{s}}}^{1 / \gamma^{\theta_{2}}}\left(e^{i \phi(u)}-e^{i u^{\alpha_{s}}}\right) \sin \psi(u) \frac{d u}{u}+\int_{1 / \gamma^{\theta_{2}}}^{1 / \gamma^{\theta}} e^{i \phi(u)} \sin \psi(u) \frac{d u}{u} \\
& -\int_{1 / \gamma^{\theta_{2}}}^{1 / \gamma^{\theta}} e^{i u^{\alpha_{s}}} \sin \psi(u) \frac{d u}{u} \\
:= & G(\gamma)+H(\gamma)-I(\gamma) .
\end{aligned}
$$

Now for $G(\gamma)$ if we follow the same steps as for $F(\gamma)$ in the previous case $\left(\theta_{1}>\theta\right)$, then we get

$$
|G(\gamma)| \leq c \gamma^{k+1-\frac{\alpha_{s-1}}{\alpha_{s}}-\theta_{2}\left(\alpha_{s-1}+\beta_{t}\right)} .
$$

Since $\theta_{2}<\theta_{1}$, we see that $G(\gamma)$ is an error term.

We now consider $H(\gamma)$. We know that if $\gamma$ is sufficiently small and $u$ is sufficiently large, then

$$
\phi^{\prime}(u) \geq c u^{\alpha_{s}-1}
$$

Also if $\Psi(u)=\sin \psi(u)$, then

$$
\Psi^{\prime}(u)=O\left(\gamma^{1-\frac{\beta_{t}}{\alpha_{s}}} u^{\beta_{t}-1}\right) \quad \text { for } u \geq \gamma^{1 / \alpha_{s}}
$$

and so integration by parts implies that $H(\gamma)=O\left(\gamma^{k+\epsilon}\right)$ for some $\epsilon>0$. In short, $H(\gamma)$ is an error term and exactly the same proof works to show that $I(\gamma)$ is an error term. So we are just left with $D(\gamma)$ that will give us the principal term. Now

$$
\begin{aligned}
D(\gamma)= & \int_{0}^{1 / \gamma^{\theta}} e^{i u^{\alpha_{s}}}(\sin \psi(u)-\psi(u)) \frac{d u}{u}+\int_{0}^{1 / \gamma^{\theta}} e^{i u^{\alpha_{s}}}\left(\gamma^{1-\frac{\beta_{1}}{\alpha_{s}}} u^{\beta_{1}}\right) \frac{d u}{u} \\
& +\int_{0}^{1 / \gamma^{\theta}} e^{i u^{\alpha_{s}}}\left(\gamma^{1-\frac{\beta_{2}}{\alpha_{s}}} u^{\beta_{2}}\right) \frac{d u}{u}+\cdots+\int_{0}^{1 / \gamma^{\theta}} e^{i u^{\alpha_{s}}}\left(\gamma^{1-\frac{\beta_{t}}{\alpha_{s}}} u^{\beta_{t}}\right) \frac{d u}{u} \\
:= & J(\gamma)+K_{1}(\gamma)+K_{2}(\gamma)+\cdots+K_{t}(\gamma) .
\end{aligned}
$$


We first consider $K_{t}(\gamma)$ :

$$
\begin{aligned}
K_{t}(\gamma) & =\gamma^{k} \int_{0}^{\infty} e^{i u^{\alpha_{s}}} u^{\beta_{t}-1} d u-\gamma^{k} \int_{1 / \gamma^{\theta}}^{\infty} e^{i u^{\alpha_{s}}} u^{\beta_{t}-1} d u \\
& :=L_{t}(\gamma)-N_{t}(\gamma)
\end{aligned}
$$

Now for $1 \leq j \leq t$, define

$$
c(j)=\int_{0}^{\infty} e^{i u^{\alpha_{s}}} u^{\beta_{j}-1} d u .
$$

We can evaluate this integral explicitly using the techniques from complex analysis. In fact,

$$
c(j)=\frac{1}{\alpha_{s}} e^{\frac{i \pi}{2 \alpha_{s}} \beta_{j}} \Gamma\left(\frac{\beta_{j}}{\alpha_{s}}\right) \neq 0
$$

where $\Gamma$ denotes the usual Gamma function. We now have $L_{t}(\gamma)=c(t) \gamma^{k}$ and also it is easy to see that $N_{t}(\gamma)=O\left(\gamma^{k+\theta\left(\alpha_{s}-\beta_{t}\right)}\right)$. Thus,

$$
K_{t}(\gamma)=c(t) \gamma^{k}+O\left(\gamma^{k+\theta\left(\alpha_{s}-\beta_{t}\right)}\right)
$$

Similarly, it can be shown that for $1 \leq j \leq t-1$,

$$
K_{j}(\gamma)=c(j) \gamma^{1-\frac{\beta_{j}}{\alpha_{s}}}+O\left(\gamma^{1-\frac{\beta_{j}}{\alpha_{s}}+\theta\left(\alpha_{s}-\beta_{j}\right)}\right)
$$

But $1-\beta_{t-1} / \alpha_{s}<1-\beta_{j} / \alpha_{s}$ for $1 \leq j<t-1$ and $\alpha_{s}-\beta_{j}>0$ for all $j$. So we have

$$
D(\gamma)=J(\gamma)+c(t) \gamma^{1-\frac{\beta_{t}}{\alpha_{s}}=k}+O\left(\gamma^{1-\frac{\beta_{t-1}}{\alpha_{s}}}\right) .
$$

Thus, we now just need to show that $J(\gamma)$ is an error term to prove our claim. For this, we shall use the following estimates:

$$
\sin \psi(u)-\psi(u)=O\left(\gamma^{3\left(1-\frac{\beta_{1}}{\alpha_{s}}\right)} u^{3 \beta_{1}}\right) \quad \text { for } u \leq \gamma^{1 / \alpha_{s}}
$$

and

$$
\sin \psi(u)-\psi(u)=O\left(\gamma^{3\left(1-\frac{\beta_{t}}{\alpha_{s}}\right)} u^{3 \beta_{t}}\right) \quad \text { for } u \geq \gamma^{1 / \alpha_{s}}
$$

Now

$$
\begin{aligned}
J(\gamma) & =\int_{0}^{\gamma^{1 / \alpha_{s}}} e^{i u^{\alpha_{s}}}(\sin \psi(u)-\psi(u)) \frac{d u}{u}+\int_{\gamma^{1 / \alpha_{s}}}^{1 / \gamma^{\theta}} e^{i u^{\alpha_{s}}}(\sin \psi(u)-\psi(u)) \frac{d u}{u} \\
& :=P(\gamma)+Q(\gamma) .
\end{aligned}
$$

From (42), it follows that $P(\gamma)=O\left(\gamma^{3}\right)$ and so finally we consider $Q(\gamma)$. Let $\Phi(u)=$ $\sin \psi(u)-\psi(u)$. Then, (43) is same as

$$
\Phi(u)=O\left(\gamma^{3 k} u^{3 \beta_{t}}\right) \quad \text { for } u \geq \gamma^{1 / \alpha_{s}} .
$$


Also $\Phi^{\prime}(u)=O\left(\gamma^{3 k} u^{3 \beta_{t}-1}\right)$. If we now integrate by parts and use the estimates of $\Phi(u)$ and $\Phi^{\prime}(u)$, then it follows that $Q(\gamma)$ is an error term. Thus,

$$
M(\gamma)=c(t) \gamma^{k}+O\left(\gamma^{l}\right) \quad \text { with } l>k .
$$

This concludes our claim for $M(\gamma)$ and also completes the proof of our main theorem.

Acknowledgements. The author would like to thank Prof. Anthony Carbery and Prof. James Wright for their useful suggestions.

\section{REFERENCES}

1. A. Carbery, M. Christ and J. Wright, Multidimensional van der Corput and sublevel set estimates, J. Amer. Math. Soc. 12(4) (1999), 981-1015.

2. A. Carbery, S. Wainger and J. Wright, Double Hilbert transforms along polynomial surfaces in $\mathbf{R}^{3}$, Duke. Math. J. 101(3) (2000), 499-513.

3. J. Duoandikoetxea and J. L. Rubio de Francia, Maximal and singular integral operators via Fourier transform estimates, Invent. Math. 84 (1986), 541-561.

4. F. Ricci and E. M. Stein, Multiparameter singular integrals and maximal functions, Ann. Inst. Fourier (Grenoble) 42 (1992), 637-670.

5. E. M. Stein, Harmonic analysis: Real-variable methods, orthogonality, and oscillatory integrals (Princeton University Press, Princeton, NJ, 1993). 